\title{
A hybrid finite-difference and integral-equation method for modeling and inversion of marine controlled-source electromagnetic data
}

\author{
Daeung Yoon ${ }^{1}$, Michael S. Zhdanov ${ }^{1}$, Johan Mattsson ${ }^{2}$, Hongzhu Cai ${ }^{1}$, and Alexander Gribenko ${ }^{1}$
}

\begin{abstract}
One of the major problems in the modeling and inversion of marine controlled-source electromagnetic (CSEM) data is related to the need for accurate representation of very complex geoelectrical models typical for marine environment. At the same time, the corresponding forward-modeling algorithms should be powerful and fast enough to be suitable for repeated use in hundreds of iterations of the inversion and for multiple transmitter/receiver positions. To this end, we have developed a novel 3D modeling and inversion approach, which combines the advantages of the finitedifference (FD) and integral-equation (IE) methods. In the framework of this approach, we have solved Maxwell's equations for anomalous electric fields using the FD approximation on a staggered grid. Once the unknown electric fields in the computation domain of the FD method are computed, the electric and magnetic
\end{abstract}

fields at the receivers are calculated using the IE method with the corresponding Green's tensor for the background conductivity model. This approach makes it possible to compute the fields at the receivers accurately without the need of very fine FD discretization in the vicinity of the receivers and sources and without the need for numerical differentiation and interpolation. We have also developed an algorithm for 3D inversion based on the hybrid FD-IE method. In the case of the marine CSEM problem with multiple transmitters and receivers, the forward modeling and the Fréchet derivative calculations are very time consuming and require using large memory to store the intermediate results. To overcome those problems, we have applied the moving sensitivity domain approach to our inversion. A case study for the 3D inversion of towed streamer EM data collected by PGS over the Troll field in the North Sea demonstrated the effectiveness of the developed hybrid method.

\section{INTRODUCTION}

The main engine for improvement in the interpretation of geophysical electromagnetic (EM) survey data is the continuing development of efficient algorithms for 3D EM modeling and inversion. There are several popular numerical approaches for 3D EM modeling: integral-equation (IE), finite-difference (FD), and finiteelement (FE) methods (Zhdanov, 2002, 2009, 2015; Avdeev, 2005).

The IE method represents one of the most effective numerical solvers for localized anomalous structures embedded in a layered earth. One of the advantages of the IE method is that it only requires a solution within the anomalous domain, and the electric and magnetic fields at the receivers are calculated based on Green's tensor approach. The IE modeling domain includes inhomogeneous geoelectrical structures only, and it is typically very small compared to the modeling domains of the differential-equation (DE) methods, which require a large computational domain to satisfy to the corresponding boundary conditions. At the same time, the system matrix of the IE method is dense, so if the complexity of the model grows, the IE method requires a significantly larger amount of computational memory and time. We should note, however, that many novel approaches have been applied to the conventional IE method to overcome those computational difficulties, including the contraction IE method, variable background conductivity, and efficient Green's tensor calculations (e.g., Hursán and Zhdanov, 2002; Zhdanov et al., 2006; Endo et al., 2008; Avdeev and Knizhnik, 2009; Zhdanov, 2009).

The advantage of the DE method is the sparsity of the system matrices, which improves the condition number and enables us to use a direct solver very efficiently. Direct solvers have tradition-

\footnotetext{
Manuscript received by the Editor 24 September 2015; revised manuscript received 29 April 2016; published online 27 July 2016.

${ }^{1}$ Consortium for Electromagnetic Modeling and Inversion, University of Utah, Salt Lake City, Utah and TechnoImaging, Salt Lake City, Utah. E-mail: duyoon@gmail.com; michael.s.zhdanov@gmail.com; caihongzhu@hotmail.com; alexgribenko@gmail.com.

${ }^{2}$ Petroleum Geo-Services, Oslo, Norway. E-mail: Johan.Mattsson@pgs.com.

(C) 2016 Society of Exploration Geophysicists. All rights reserved.
} 
ally been considered to be too computationally demanding for 3D problems compared to iterative solvers. However, gradual advancement of direct-solution algorithms, along with the availability of resources for parallel computation, makes it possible to apply these algorithms for solving large-scale 3D problems very efficiently (Streich, 2009; da Silva et al., 2012; Yang and Oldenburg, 2012; Grayver et al., 2013; Schwarzbach and Haber, 2013; Jaysaval et al., 2014). In particular, in the case of multisource problems such as marine CSEM, the direct solvers may perform more efficiently than the iterative solvers because the direct solvers reuse the decomposed system matrix for multiple sources whereas the iterative solvers need to solve the problem for each source separately (Chung et al., 2014). However, DE methods require a very large computational domain and extensive mesh refinement in the vicinity of the receivers and/or sources to reduce errors caused by the interpolation and numerical differentiation required to calculate the electric and magnetic fields in the receivers. To avoid mesh refinement and/or numerical errors, Cox and Zhdanov (2014) apply the Green's tensor approach to the FE method to calculate magnetic fields and their sensitivities at the receivers, and develop a 3D airborne EM inversion based on their hybrid FE-IE method. In this paper, we use a concept similar to the Green's tensor approach and apply it to the FD method.

We have developed a hybrid FD-IE method, which combines the advantages of those two methods. In the framework of this approach, the FD modeling algorithm is based on the staggered grid (Yee, 1966) and follows the approach outlined in Newman and Alumbaugh (1995). Once the unknown electric fields in the computation domain of the FD method are computed, the electric and magnetic fields at the receivers are calculated using the IE method with the corresponding Green's tensor for the background conductivity model. This approach makes it possible to compute the fields at the receivers accurately without the need of very fine FD discretization in the vicinity of the receivers and transmitters and without the need for numerical differentiation and interpolation. We should note that recently, Zaslavsky et al. (2011) developed a novel version of the hybrid FD-IE method for solving 3D frequency-domain anisotropic electromagnetic problems. However, the main focus of the cited paper is on the construction of an effective preconditioning operator for their FD solver using a volume IE approach. In our paper, we apply a multifrontal massively parallel sparse (MUMPS) direct solver to solve the system of FD equations. This enables an efficient solution of large-scale problems with multiple sources.

To verify the accuracy of the developed method, we compare the fields computed by the hybrid FD-IE method with those computed by the conventional FD method, the 1D semianalytical solution, and the 3D IE method.

The developed hybrid algorithm was incorporated as the forward EM modeling engine in a general regularized inversion scheme, based on the reweighted conjugate gradient method. One of the key elements of our inversion algorithm is computing the Fréchet derivative matrix. A conventional approach to solving this problem is based on using the adjoint operator (e.g., Avdeev, 2005; Abubakar et al., 2011; Zhdanov, 2002, 2015). However, in a case of a large-scale problem, typical for a towed streamer EM method, when the survey covers hundreds and even thousands of line kilometers, this approach becomes impractical. To reduce the memory requirements, we apply the moving sensitivity domain (MSD) approach
(Zhdanov et al., 2014a, 2014b). The MSD technique, critical for this large-scale problem, requires assembling a "global" sensitivity matrix for entire inversion domain corresponding to the total survey area. The inversion runs simultaneously for this large survey, but the sensitivity matrix becomes very sparse due to the MSD approach, which dramatically reduces the memory requirements and makes large-scale inversion feasible. In the developed inversion algorithm, the Fréchet derivative matrix is calculated using a quasi-Born (QB) approximation (Gribenko and Zhdanov, 2007; Zhdanov, 2009). The QB approximation provides the necessary tool for solving this problem because it gives a quasianalytical representation for the Fréchet derivative. Remarkably, using the QB approximation does not slow the convergence of inversion, as was demonstrated in a number of publications on EM inversion using the IE method (e.g., Gribenko and Zhdanov, 2007; Zhdanov et al., 2014a, 2014b). The QB approximation is extremely effective in the framework of the IE method because the Green's functions are already precomputed, and QB approximation requires just one additional matrix multiplication operation. The beauty of the hybrid FD-IE approach is that in the framework of this method, we also have Green's functions precomputed and QB approximation comes practically for free.

In summary, there are three advantages of the developed inversion scheme: (1) the data predicted by the hybrid FD-IE method provide a more accurate solution than the conventional FD method, even without the mesh refinement near the receiver and source positions; (2) the QB approximation enables us to calculate the Fréchet derivative matrix very efficiently without any extra forward modeling because Green's tensors used for the EM field calculation in the forward modeling are reused for the Fréchet derivative calculation, so no extra computation of Green's tensors is required; and (3) the memory requirements for storing the intermediate forward modeling results and the Fréchet derivative matrix are reduced due to the application of the MSD approach.

Although the inversion algorithm is general, this paper presents an application of this method specifically to the 3D inversion of towed streamer EM data. A model study of the 3D inversion of synthetic towed streamer EM data is presented to demonstrate the effectiveness of the developed hybrid method. We have also applied the novel 3D inversion method to the towed streamer EM data collected by PGS in the Troll West Oil Province (TWOP; Zhdanov et al., 2014b).

\section{FORMULATION OF A HYBRID FD-IE METHOD}

\section{FD modeling of the anomalous electric field}

The implementation of the FD method developed in this paper follows that of Newman and Alumbaugh (1995) and Alumbaugh et al. (1996). The method solves Maxwell's equations in the frequency domain based on an FD scheme on a staggered grid and uses the anomalous field formulation with the total field being decomposed into background $\mathbf{E}^{\mathrm{b}}$ and anomalous $\mathbf{E}^{\mathrm{a}}$ fields. The calculation of the anomalous field with an equivalent source makes it possible to avoid the discretization problems associated with discrete sources. This approach has been widely used in the EM modeling literature, whether with FD, finite-volume, FE, or IE methods (e.g., Zhdanov and Keller, 1994; Zhdanov, 2002, 2009).

We should note that the approach based on a decomposition of the total EM field into separate background and anomalous fields plays a very important role in the developed hybrid FD-IE method. 
First, assuming that the background field is known, we can apply the FD modeling for the anomalous field only. As a result, we avoid the additional complexity of FD modeling of the source field because the background field is assumed to be known. Second, by reducing the FD modeling to the anomalous field only, we can dramatically reduce the modeling domain because the anomalous field attenuates much faster than the total field. Third, the background field is required in the IE formulation of the forward modeling problem; it is a common practice to select the background field in such a way that it could be relatively easily found. The conventional approach is based on using as a background field the EM field generated in the horizontally layered medium.

For the low-frequency EM fields considered in geophysical applications the displacement currents can be ignored in Maxwell's equations, which results in the second-order DE for the anomalous electric field $\mathbf{E}^{\mathrm{a}}$ :

$$
\nabla \times \nabla \times \mathbf{E}^{\mathrm{a}}+i \omega \mu_{0} \boldsymbol{\sigma} \mathbf{E}^{\mathrm{a}}=-i \omega \mu_{0}\left(\boldsymbol{\sigma}-\boldsymbol{\sigma}^{\mathrm{b}}\right) \mathbf{E}^{\mathrm{b}}
$$

The magnetic permeability within the earth $\mu$ is assumed to be constant, $\mu_{0}=4 \pi \times 10^{-7} \mathrm{H} / \mathrm{m}$, and the total $\sigma$, and background $\boldsymbol{\sigma}^{\mathrm{b}}$ conductivity tensors are considered to be diagonal, composed of the principal components of the conductivity tensor. The electric fields are assigned to the edges of the cells in the staggered grid. For simplicity, we use the Dirichlet boundary condition; i.e., all components of the anomalous electric field are set to zero at all sides of the computational domain. This is justified because in marine environment, the anomalous field attenuates fast enough in the conductive water. Therefore, at some large enough distance of the boundaries from the anomalous domain, we may neglect the values of the anomalous field at the boundaries (including those located above the sea level in the air). However, in a general case, a more accurate boundary condition can be used (e.g., Zhdanov, 2002; Avdeev, 2005; Newman, 2014).

An FD representation of equation 1 on a staggered grid can be written as a linear system of equations as follows:

$$
\mathbf{K e}=\mathbf{R},
$$

where $\mathbf{e}$ is the unknown vector of the anomalous electric field and $\mathbf{R}$ is a vector containing the source terms. The matrix $\mathbf{K}$ is a sparse and symmetric complex matrix composed of real numbers except for the diagonal elements. We use MUMPS direct solver (Amestoy et al., 2001,2006 ) to solve the system of equation 2, which enables an efficient solution of large-scale problems with multiple sources.

\section{IE method for computing the EM field at the receivers}

The conventional FD method requires an interpolation to calculate the electric fields at the receivers, a numerical approximation of the curl of the electric field, and an interpolation to calculate the magnetic fields at the receivers. Such numerical differentiations and interpolations can cause some numerical errors and require mesh refinement in the vicinity of the receivers to reduce the errors. To avoid those problems, we use an IE approach to calculate the electric and magnetic fields at the receivers. The anomalous electric and magnetic fields at the receiver position $\boldsymbol{r}_{l}, l=1,2, \ldots, L$ can be expressed as an integral over the excess currents in the anomalous domain $D$ :

$$
\begin{aligned}
& \mathbf{E}^{\mathrm{a}}\left(\boldsymbol{r}_{l}\right)=\iiint_{D} \hat{\boldsymbol{G}}_{\mathbf{E}}\left(\boldsymbol{r}_{l} \mid \boldsymbol{r}\right) \cdot\left[\Delta \boldsymbol{\sigma}(\boldsymbol{r}) \cdot\left[\mathbf{E}^{\mathrm{b}}(\boldsymbol{r})+\mathbf{E}^{\mathrm{a}}(\boldsymbol{r})\right]\right] d v, \\
& \mathbf{H}^{\mathrm{a}}\left(\boldsymbol{r}_{l}\right)=\iiint_{D} \hat{\boldsymbol{G}}_{\mathbf{H}}\left(\boldsymbol{r}_{l} \mid \boldsymbol{r}\right) \cdot\left[\Delta \boldsymbol{\sigma}(\boldsymbol{r}) \cdot\left[\mathbf{E}^{\mathrm{b}}(\boldsymbol{r})+\mathbf{E}^{\mathrm{a}}(\boldsymbol{r})\right]\right] d v,
\end{aligned}
$$

where $\hat{\boldsymbol{G}}_{\mathbf{E}}\left(\boldsymbol{r}_{l} \mid \boldsymbol{r}\right)$ and $\hat{\boldsymbol{G}}_{\mathbf{H}}\left(\boldsymbol{r}_{l} \mid \boldsymbol{r}\right)$ are the electric and magnetic Green's tensors defined for an unbounded conductive medium with the background (horizontally layered) anisotropic conductivity $\boldsymbol{\sigma}^{\mathrm{b}}$; the anomalous domain $D$ represents a volume with the anisotropic anomalous conductivity distribution $\Delta \boldsymbol{\sigma}(\boldsymbol{r})=\boldsymbol{\sigma}(\boldsymbol{r})-\boldsymbol{\sigma}^{b}(\boldsymbol{r})$.

Note that, in the case of the IE method, we use the node-based grid with vector $\boldsymbol{r}$ located at the center of the cell, in which all three components of the electric fields are assigned. However, in the FD scheme based on the staggered grid, the electric fields to be solved are located at the edges of the cells. Therefore, to use the IE method, we need to adjust either the electric fields on the staggered grid to the center of the cell or the Green's tensor itself for the staggered grid. Thus, one can introduce two different ways to use the IE formulation to compute the fields at the receiver positions. The first approach uses the interpolation of the electric field in the center of the cell. The second is based on computing the Green's tensors directly on the staggered grid without any interpolation.

We have tested both approaches to the solution of this problem. In the case of the first approach, interpolation of electric fields form the edges of the cell to its center, we have used the scheme introduced in Streich (2009). As discussed above, the second approach is based on computing the Green's tensors on the staggered grid without any interpolation. We will present a discrete form of equation 3 for this situation below. Obviously, the hybrid FD-IE method based on this second approach requires additional computations of the Green's tensors in comparison with the first approach, discussed above, because the Green's tensor has to be calculated not only in the centers of each cells, but also in the centers of every edge as well. However, it is preferable to use this approach in the framework of the hybrid FD-IE method because in this case one can avoid the interpolation and differentiation errors or refinement near receivers to enhance the accuracy of the field calculation. The results of our numerical study have confirmed that, indeed, the second approach provides a more accurate result. That is why we use the second approach in our inversion algorithm as well.

In discrete form, we define the main node $(i, j, k)$ to be located at the center of the cells in the inhomogeneous domain $D$ and denote the total number of cells in domain $D$ as follows: $N=N_{x} \times$ $N_{y} \times N_{z}$. The total number of edges of the cells is calculated as follows: $N^{e}=N_{x}^{e}+N_{y}^{e}+N_{z}^{e}$, where $N_{x}^{e}=N_{x} \times\left(N_{y}+1\right) \times$ $\left(N_{z}+1\right), N_{y}^{e}=\left(N_{x}+1\right) \times N_{y} \times\left(N_{z}+1\right)$, and $N_{y}^{e}=\left(N_{x}+1\right) \times$ $\left(N_{y}+1\right) \times N_{z}$.

In the case of node-based grid with three-directional anisotropic conductivity, equation 3 can be simplified using matrix notations as follows (Hursán and Zhdanov, 2002):

$$
\mathbf{E}^{\mathrm{a}}=\hat{\boldsymbol{G}}^{\mathrm{E}} \Delta \boldsymbol{\sigma} \mathbf{E},
$$

where $\mathbf{E}^{\mathrm{a}}$ is a $3 L \times 1$ vector column of $x$-, $y$-, and $z$-components of anomalous electric fields at the receiver positions $\boldsymbol{r}_{l}, l=$ $1,2, \ldots, L$, 


$$
\mathbf{E}^{\mathrm{a}}=\left[\mathrm{E}_{x, 1}^{\mathrm{a}}, \ldots, \mathrm{E}_{x, L}^{\mathrm{a}}, \mathrm{E}_{y, 1}^{\mathrm{a}}, \ldots, \mathrm{E}_{y, L}^{\mathrm{a}}, \mathrm{E}_{z, 1}^{\mathrm{a}}, \ldots, \mathrm{E}_{z, L}^{\mathrm{a}}\right]^{\mathrm{T}},
$$

and $\mathbf{E}$ is a $3 N \times 1$ vector column of electric fields at the centers of the cells within the inhomogeneous domain $D$ :

$$
\begin{gathered}
\mathbf{E}=\left[\mathrm{E}_{x,(1,1,1)}, \ldots, \mathrm{E}_{x,\left(N_{x}, N_{y}, N_{z}\right)}, \mathrm{E}_{y,(1,1,1)}, \ldots, \mathrm{E}_{y,\left(N_{x}, N_{y}, N_{z}\right)},\right. \\
\left.\mathrm{E}_{z,(1,1,1)}, \ldots, \mathrm{E}_{z,\left(N_{x}, N_{y}, N_{z}\right)}\right]^{\mathrm{T}} .
\end{gathered}
$$

Matrix $\hat{\boldsymbol{G}}^{\mathrm{E}}$ is a $3 L \times 3 N$ rectangular matrix containing the integrals of the electric Green's tensors,

$$
\begin{gathered}
\hat{\boldsymbol{G}}^{\mathrm{E}}=\left[\begin{array}{lll}
\boldsymbol{G}_{x x}^{\mathrm{E}} & \boldsymbol{G}_{x y}^{\mathrm{E}} & \boldsymbol{G}_{x z}^{\mathrm{E}} \\
\boldsymbol{G}_{y x}^{\mathrm{E}} & \boldsymbol{G}_{y y}^{\mathrm{E}} & \boldsymbol{G}_{y z}^{\mathrm{E}} \\
\boldsymbol{G}_{z x}^{\mathrm{E}} & \boldsymbol{G}_{z y}^{\mathrm{E}} & \boldsymbol{G}_{z z}^{\mathrm{E}}
\end{array}\right] \\
\boldsymbol{G}_{\alpha \beta}^{\mathrm{E}}=\left[\begin{array}{ccc}
\Gamma_{\alpha \beta}^{1(1,1,1)} & \cdots & \Gamma_{\alpha \beta}^{1\left(N_{x}, N_{y}, N_{z}\right)} \\
\vdots & \ddots & \vdots \\
\Gamma_{\alpha \beta}^{L(1,1,1)} & \cdots & \Gamma_{\alpha \beta}^{L\left(N_{x}, N_{y}, N_{z}\right)}
\end{array}\right], \quad \alpha, \beta=x, y, z, \\
\Gamma_{\alpha \beta}^{l(i, j, k)}= \\
\iint_{D_{(i, j, k)}} G_{\alpha \beta}^{\mathrm{E}}\left(r_{l} \mid r_{(i, j, k)}\right) d v
\end{gathered}
$$

where $G_{\alpha \beta}^{\mathrm{E}}\left(r_{l} \mid r_{(i, j, k)}\right), \alpha=x, y, z$, are the components of the electric Green's tensors; $\Delta \boldsymbol{\sigma}$ is a $3 N \times 3 N$ diagonal matrix with anomalous conductivities; and

$$
\begin{gathered}
\Delta \boldsymbol{\sigma}=\operatorname{diag}\left(\left[\Delta \sigma_{x(1,1,1)}, \ldots, \Delta \sigma_{x\left(N_{x}, N_{y}, N_{z}\right)}, \Delta \sigma_{y(1,1,1)}, \ldots,\right.\right. \\
\left.\left.\Delta \sigma_{y\left(N_{x}, N_{y}, N_{z}\right)}, \Delta \sigma_{z(1,1,1)}, \ldots, \Delta \sigma_{z\left(N_{x}, N_{y}, N_{z}\right)}\right]\right) .
\end{gathered}
$$

Note that $r_{(i, j, k)}$ in equation 10 is located at the center of the cell where the electric field is computed using the IE method. However, as we discussed above, in the framework of the FD scheme based on the staggered grid, the electric fields are calculated at the edges of the cells. Therefore, to apply the IE formulation in equation 3 to the FD scheme on the staggered grid, equation 5 should be modified accordingly as follows:

$$
\mathbf{E}^{\mathrm{a}}=\hat{\mathbf{G}}^{\mathrm{E}} \Delta \boldsymbol{\sigma}^{\prime} \mathbf{E}^{\prime}
$$

where "prime" indicates that the components of all matrices in equation 12 are given at the midpoints of the edges of the corresponding staggered grid, as shown below.

Matrix $\Delta \boldsymbol{\sigma}^{\prime}$ is an $N^{e} \times N^{e}$ diagonal matrix of the weighted averaging anomalous conductivities, defined in Appendix A,

$$
\begin{gathered}
\Delta \boldsymbol{\sigma}^{\prime}=\operatorname{diag}\left(\left[\Delta \sigma_{x\left(1, \frac{1}{2}, \frac{1}{2}\right)}, \ldots, \Delta \sigma_{x\left(N_{x}, N_{y}+\frac{1}{2}, N_{z}+\frac{1}{2}\right)}, \Delta \sigma_{y\left(\frac{1}{2}, 1, \frac{1}{2}\right)}, \ldots,\right.\right. \\
\Delta \sigma_{y\left(N_{x}+\frac{1}{2}, N_{y}, N_{z}+\frac{1}{2}\right)}, \Delta \sigma_{z\left(\frac{1}{2}, \frac{1}{2}, 1\right)}, \ldots, \Delta \sigma_{\left.\left.z\left(N_{x}+\frac{1}{2}, N_{y}+\frac{1}{2}, N_{z}\right)\right]\right) .}
\end{gathered}
$$

Vector $\mathbf{E}^{\prime}$ is an $N^{e} \times 1$ vector column of electric fields within the inhomogeneous domain $D$,

$$
\begin{aligned}
\mathbf{E}^{\prime}= & {\left[\mathrm{E}_{x,\left(1, \frac{1}{2}, \frac{1}{2}\right)}, \ldots, \mathrm{E}_{x,\left(N_{x}, N_{y}+\frac{1}{2}, N_{z}+\frac{1}{2}\right)}, \mathrm{E}_{y,\left(\frac{1}{2}, 1, \frac{1}{2}\right)}, \ldots,\right.} \\
& \left.\mathrm{E}_{y,\left(N_{x}+\frac{1}{2}, N_{y}, N_{z}+\frac{1}{2}\right)}, \mathrm{E}_{z,\left(\frac{1}{2}, \frac{1}{2}, 1\right)}, \ldots, \mathrm{E}_{z,\left(N_{x}+\frac{1}{2}, N_{y}+\frac{1}{2}, N_{z}\right)}\right]^{\mathrm{T}} .
\end{aligned}
$$

Matrix $\hat{\mathbf{G}}^{\mathrm{E}}$ is a $3 L \times N^{e}$ rectangular matrix containing the integrals of the electric Green's tensors, defined according to the following formula:

$$
\hat{\mathbf{G}}^{\prime \mathrm{E}}=\left[\begin{array}{lll}
\mathbf{G}_{x x}^{\prime \mathrm{E}} & \mathbf{G}_{x y}^{\prime \mathrm{E}} & \mathbf{G}_{x z}^{\prime \mathrm{E}} \\
\mathbf{G}_{y x}^{\prime \mathrm{E}} & \mathbf{G}_{y y}^{\prime \mathrm{E}} & \mathbf{G}_{y z}^{\prime \mathrm{E}} \\
\mathbf{G}_{z x}^{\prime \mathrm{E}} & \mathbf{G}_{z y}^{\prime \mathrm{E}} & \mathbf{G}_{z z}^{\prime \mathrm{E}}
\end{array}\right]
$$

In the last formula, $\mathbf{G}_{\alpha x}^{\mathrm{E}}$ is an $L \times N_{x}^{e}$ matrix,

$$
\mathbf{G}_{\alpha x}^{\mathrm{E}}=\left[\begin{array}{ccc}
\Gamma_{\alpha x}^{1\left(1, \frac{1}{2}, \frac{1}{2}\right)} & \cdots & \Gamma_{\alpha x}^{1\left(N_{x}, N_{y}+\frac{1}{2}, N_{z}+\frac{1}{2}\right)} \\
\vdots & \ddots & \vdots \\
\Gamma_{\alpha x}^{L\left(1, \frac{1}{2}, \frac{1}{2}\right)} & \cdots & \Gamma_{\alpha x}^{L\left(N_{x}, N_{y}+\frac{1}{2}, N_{z}+\frac{1}{2}\right)}
\end{array}\right]
$$

$\mathbf{G}_{\alpha y}^{\prime \mathrm{E}}$ is an $L \times N_{y}^{e}$ matrix,

$$
\mathbf{G}_{\alpha y}^{\mathrm{E}}=\left[\begin{array}{ccc}
\Gamma_{\alpha y}^{1\left(\frac{1}{2}, 1, \frac{1}{2}\right)} & \ldots & \Gamma_{\alpha y}^{1\left(N_{x}+\frac{1}{2}, N_{y}, N_{z}+\frac{1}{2}\right)} \\
\vdots & \ddots & \vdots \\
\Gamma_{\alpha y}^{L\left(\frac{1}{2}, 1, \frac{1}{2}\right)} & \cdots & \Gamma_{\alpha y}^{L\left(N_{x}+\frac{1}{2}, N_{y}, N_{z}+\frac{1}{2}\right)}
\end{array}\right],
$$

and $\mathbf{G}_{\alpha z}^{\prime \mathrm{E}}$ is an $L \times N_{z}^{e}$ matrix:

$$
\mathbf{G}_{\alpha z}^{\prime \mathrm{E}}=\left[\begin{array}{ccc}
\Gamma_{\alpha z}^{1\left(\frac{1}{2}, \frac{1}{2}, 1\right)} & \cdots & \Gamma_{\alpha z}^{1\left(N_{x}+\frac{1}{2}, N_{y}+\frac{1}{2}, N_{z}\right)} \\
\vdots & \ddots & \vdots \\
\Gamma_{\alpha z}^{L\left(\frac{1}{2}, \frac{1}{2}, 1\right)} & \cdots & \Gamma_{\alpha z}^{L\left(N_{x}+\frac{1}{2}, N_{y}+\frac{1}{2}, N_{z}\right)}
\end{array}\right]
$$

Where

$$
\Gamma_{\alpha \beta}^{l(i, j, k)}=\iiint_{D_{(i, j, k)}} G_{\alpha \beta}^{\mathrm{E}}\left(r_{l} \mid r_{(i, j, k)}\right) d v
$$

Magnetic fields at the receiver positions can be calculated in a similar way, by replacing the magnetic Green's tensors in equation 19 for the electric Green's tensors.

The hybrid FD-IE method makes it possible to avoid the interpolation and differentiation errors and/or refinement of the grid in the vicinity of the receivers to improve the accuracy of the field calculation. Also, the discretization grid outside the anomalous domain can be coarser than for the conventional FD method because the hybrid method requires an accurate solution within the anomalous domain only.

Obviously, the hybrid FD-IE modeling method requires an additional computation of Green's tensors in comparison with the con- 
ventional FD modeling method. The advantage of this approach in the inversion algorithm is that the Green's tensors used for the field calculation in the receivers are the same as those used for the Fréchet derivative calculation for the EM inversion. Therefore, the precomputed Green's tensor for the field calculation in the receivers can be reused for the Fréchet derivative calculation with practically no extra computation required.

\section{INVERSION METHODOLOGY}

We have implemented the developed hybrid FD-IE modeling method in the algorithm of inversion of the marine CSEM data following the paper by Gribenko and Zhdanov (2007). The regularized inversion algorithm is based on minimization of the Tikhonov parametric functional (Tikhonov and Arsenin, 1977; Zhdanov, 2002, 2015):

$$
P(\boldsymbol{\Delta} \boldsymbol{\sigma})=\left\|\mathbf{W}_{d}\left(\mathbf{A}_{h}(\Delta \boldsymbol{\sigma})-\mathbf{d}\right)\right\|^{2}+\alpha \boldsymbol{s}(\boldsymbol{\Delta} \boldsymbol{\sigma})=\min ,
$$

where $\mathbf{d}$ is the vector of the observed data, $\mathbf{A}_{h}(\boldsymbol{\Delta} \boldsymbol{\sigma})$ is the forward modeling operator for computing the predicted data based on the hybrid FD-IE method, $\alpha$ is the regularization parameter, and $\mathbf{W}_{d}$ is the diagonal data weighting matrix formed by the inverse amplitudes of the background electric field.

The first term of the parametric functional in equation 20 represents the weighted misfit functional, and the second term is a stabilizer. There are several possible choices for the stabilizer, such as maximum smoothness (e.g., the Occam or minimum norm), or focusing stabilizers (e.g., minimum support, minimum gradient support, and minimum vertical support). One can find a detailed description of the family of different stabilizing functionals in Zhdanov (2002, 2015). In the numerical examples presented below, we use the minimum norm and the minimum vertical support stabilizing functionals.

We apply the regularized conjugate gradient (RCG) algorithm of the parametric functional minimization, summarized as follows (Zhdanov, 2002, 2015):

$$
\begin{aligned}
\mathbf{r}^{(n)}= & \mathbf{A}_{h}\left(\Delta \boldsymbol{\sigma}^{(n)}\right)-\mathbf{d}, \\
\mathbf{l}^{(n)}= & \mathbf{F}^{(n) *} \mathbf{W}_{d}^{*} \mathbf{W}_{d} \mathbf{r}^{(n)} \\
& +\alpha\left(\mathbf{W}_{m} \mathbf{W}_{e}\right)^{*}\left(\mathbf{W}_{m} \mathbf{W}_{e}\right)\left(\Delta \boldsymbol{\sigma}^{(n)}-\Delta \boldsymbol{\sigma}_{\mathrm{apr}}\right), \\
\beta^{(n)}= & \left\|\mathbf{l}^{(n)}\right\|^{2} /\left\|\mathbf{l}^{(n-1)}\right\|^{2}, \\
\tilde{\mathbf{l}}^{(n)}= & \mathbf{l}^{(n)}+\beta_{n} \tilde{\mathbf{l}}^{(n-1)}, \tilde{\mathbf{l}}^{(0)}=\mathbf{l}^{(0)}, \\
k^{(n)}= & \left(\tilde{\mathbf{l}}^{(n)}, \mathbf{l}^{(n)}\right) /\left(\left\|\mathbf{W}_{d} \mathbf{F}^{(n)} \tilde{\mathbf{l}}^{(n)}\right\|^{2}+\alpha\left\|\mathbf{W}_{m} \mathbf{W}_{e} \tilde{\mathbf{l}}^{(n)}\right\|^{2}\right), \\
\Delta \boldsymbol{\sigma}^{(n+1)}= & \Delta \boldsymbol{\sigma}^{(n)}-k^{(n)} \tilde{\mathbf{l}}^{(n)},
\end{aligned}
$$

where $\mathbf{r}^{(n)}$ is a residual at the iteration step $n, \mathbf{l}^{(n)}$ is the gradient direction, $\tilde{\mathbf{I}}^{(n)}$ is the conjugate direction, $\mathbf{F}^{(n)}$ is a Fréchet derivative matrix, $k^{(n)}$ is a length of the iteration step, and $\mathbf{W}_{m}$ is the weighting matrix of the model parameters determined based on the weighted Fréchet derivative matrix (sensitivities):

$$
\mathbf{W}_{m}=\operatorname{diag}\left(\mathbf{F}^{*} \mathbf{W}_{d}^{*} \mathbf{W}_{d} \mathbf{F}\right)^{1 / 4} .
$$

In the case of focusing stabilizers, we use the reweighted RCG (RRCG) method introduced in Zhdanov (2002, pp. 161-166). This algorithm is similar to the RCG algorithm represented by equa- tion 21. However, the inversion is conducted in the space of the weighted conductivities $\Delta \sigma \underset{w}{(n)}$, which are related to the original conductivities by the following formula:

$$
\Delta \sigma_{w}^{(n)}=\mathbf{W}_{\mathrm{m}} \mathbf{W}_{\mathrm{e} n} \Delta \sigma_{w}^{(n)},
$$

where $\mathbf{W}_{e}$ is the variable weighting matrix determined based on the types of the stabilizer. The in-depth description of the RRCG algorithm and the corresponding analytical formulas for matrix $\mathbf{W}_{e}$ can be found in the books on inversion theory by Zhdanov (2002, 2015).

Direct computation of the Fréchet derivative is very time consuming, and at least one extra forward modeling is required at every iteration, if one would use an adjoint solution for the Fréchet derivative. This approach becomes impractical for a very large survey area, typical for towed streamer EM method, when the survey covers hundreds and even thousands of square kilometers. To overcome this problem, we calculate the Fréchet derivatives based on the QB approximation (Gribenko and Zhdanov, 2007), which does not require any extra forward modeling, so that it results in a very efficient inversion method.

Note that, in our inversion algorithm, the electric fields are computed by hybrid FD-IE modeling at the centers of the edges of the cells, whereas the model parameters are assigned to the centers of the cells. Therefore, we have to derive a discrete form of the QB approximation of the Fréchet derivatives on the edges of the cells of the staggered grid (see Appendix B). The advantage of computing the Fréchet derivatives on the staggered grid is that the Green's tensors we used in the field calculation are the same as those for the Fréchet calculation. Therefore, we do not require any extra computation of the Green's tensors for the Fréchet derivative computation. We precompute the Green's tensors only once and reuse them for the Fréchet and field calculations at every iteration of the inversion method.

Another difficulty in computing the Fréchet derivative matrix is related to the size of the computer memory required for its storage. The size of the Fréchet derivative matrix is proportional to the number of EM data points times the number of the cells in the inversion domain. If the numbers of data points and cells in the inversion domain are large, the computer memory requirements may exceed the available storage size. To reduce these requirements, we apply the MSD approach to our inversion algorithm (Cox et al., 2012; Zhdanov and Cox, 2012; Zhdanov et al., 2013, 2014a, 2014b).

In the framework of the MSD approach, for a given transmitterreceiver pair, the responses and Fréchet derivatives are computed from a subdomain that encapsulates the towed EM system's sensitivity domain. The Fréchet matrix for the entire inversion domain is then constructed as the superposition of the Fréchet derivatives from all transmitter-receiver pairs over the entire inverse model (Zhdanov et al., 2014a, 2014b). This makes the originally dense matrix of the Fréchet derivative a sparse one. In this way, we can reduce the memory requirement for the Fréchet derivative calculation while retaining maximum accuracy.

\section{VERIFICATION OF THE HYBRID FD-IE MODELING METHOD}

To verify the accuracy and the efficiency of the hybrid FDIE forward modeling method, we compare it with three other 
techniques: a 1D semianalytical solution, a conventional FD method, and a 3D IE method.

\section{Comparison with a semianalytical solution}

Model 1 is a horizontally layered geoelectrical model with an isotropic resistive rectangular reservoir (Figure 1). The background is a seawater-sediment model with an air-earth interface at $z=0 \mathrm{~m}$ and a seawater depth of $1000 \mathrm{~m}$. The resistivities of air, seawater, and sediments are $10^{6}, 0.3$, and $1 \mathrm{ohm}-\mathrm{m}$, respectively. The electromagnetic field is excited by a horizontal electric dipole oriented in the $x$-direction with a moment of $1 \mathrm{Am}$ and located in the seawater with coordinates $(0,0,950 \mathrm{~m})$, which is $50 \mathrm{~m}$ above the seafloor. The frequency of the current in the transmitting dipole is $1 \mathrm{~Hz}$. The receivers are located $5 \mathrm{~m}$ above the seafloor from $-3 \mathrm{~km}$ to $3 \mathrm{~km}$ with $200 \mathrm{~m}$ spacing in the $x$-direction. To have nonzero values of all of the scalar components of the electromagnetic field, we have placed the receivers along a profile parallel to the transmitter profile, but shifted them $50 \mathrm{~m}$ in the $y$-direction (at $y=-50 \mathrm{~m}$ ). An isotropic 3D resistive rectangular reservoir with a resistivity of $100 \mathrm{ohm}-\mathrm{m}$ is embedded in the sediments from a depth of 1400 to $1500 \mathrm{~m}$ and with a size of $3 \mathrm{~km} \times 3 \mathrm{~km} \times 100 \mathrm{~m}$ in the $x$-, $y$-, and $z$-directions, respectively, which makes it possible, based on the skin depth for this model, to approximate this reservoir accurately enough by an infinite horizontal resistive layer. The volume of the 3D resistive reservoir is considered as a domain with anomalous conductivity (anomalous domain).

To demonstrate the accuracy and efficiency of the developed hybrid FD-IE method over the conventional FD method, we calculated the EM responses for model 1 using coarse and fine grids. The responses obtained by conventional FD and hybrid FD-IE methods were then compared with the $1 \mathrm{D}$ semianalytical solution. For all
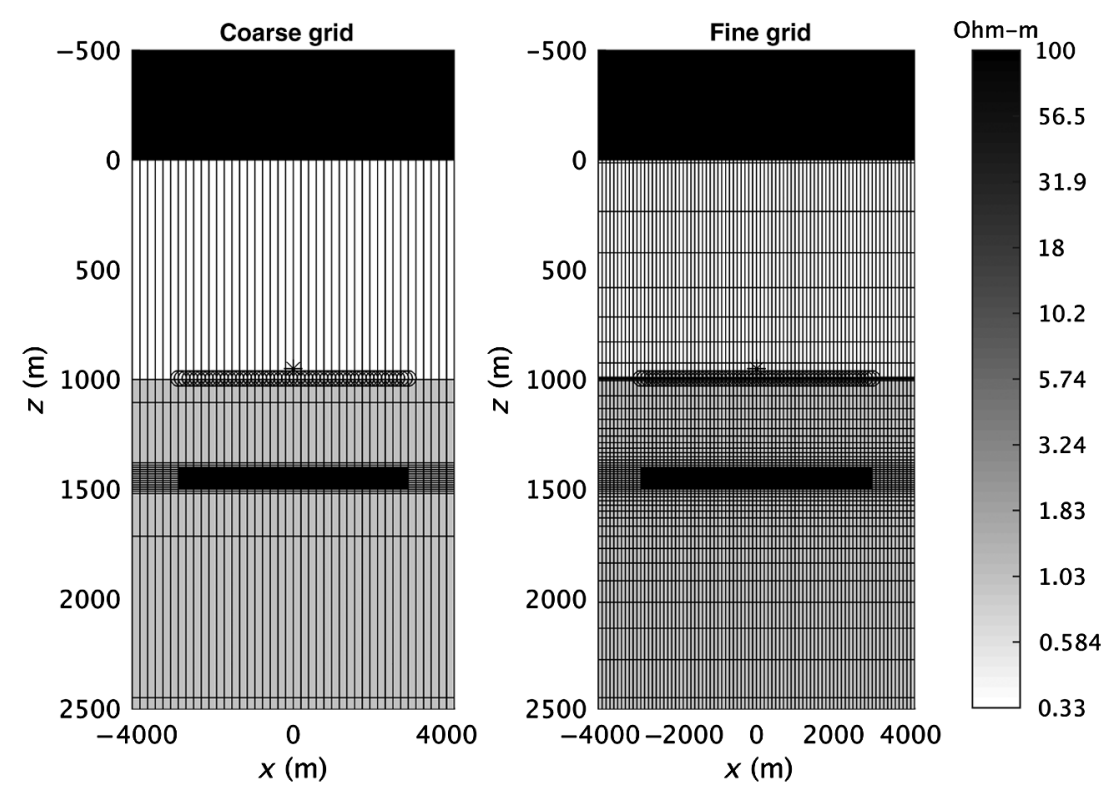

Figure 1. Model 1: A horizontally layered geoelectrical model with an isotropic reservoir approximated by an infinite horizontal resistive layer. The left panel shows a coarse grid, whereas the right panel presents a fine grid. The isotropic resistive reservoir with a resistivity of $100 \mathrm{ohm}-\mathrm{m}$ is embedded in the sediments below the seawater layer. The star indicates the position of the electrical dipole source, and the circles denote the receiver positions. grids, the FD modeling domain was selected based on the skin depth as follows: $\{-4 \leq x, y \leq 4 \mathrm{~km} ;-0.5 \leq z \leq 3 \mathrm{~km}\}$. The coarse grid consisted of $41 \times 41 \times 25=31,775$ cells, with uniform cell size of $200 \times 200 \mathrm{~m}$ in the $x$ - and $y$-directions, and logarithmically increasing cell size from the boundaries of the reservoir (anomalous domain) to the boundaries of the FD domain in the $z$-direction, as shown in the left panel of Figure 1 . The anomalous domain was discretized using a $200 \times 200 \times 10 \mathrm{~m}$ uniform grid. The anomalous electric and magnetic fields were computed by the conventional FD and hybrid FD-IE methods on different grids (see Figures 2 and 3). In the FD-only approach, the anomalous fields were linearly interpolated to the receiver points. These fields were compared with the 1D semianalytical solutions based on the Hankel transform (Ward and Hohmann, 1988; Zhdanov and Keller, 1994).

We have also calculated the relative errors $e$ of the FD modeling results using the following formula:

$$
e=\left\|\mathbf{E}_{\mathrm{san}}^{a}-\mathbf{E}^{a}\right\| /\left\|\mathbf{E}_{\mathrm{san}}^{a}\right\| \times 100,
$$

where $\mathbf{E}_{\mathrm{san}}^{\mathrm{a}}$ is anomalous electric field based on semianalytical solution, and $\mathbf{E}^{\mathrm{a}}$ is anomalous electric field based on FD and/or hybrid FD-IE solutions. A comparison of the relative errors and the corresponding computational time is given in Table 1.

As one could see, in a case of the coarse grid, the hybrid FD-IE responses are in a good agreement with the semianalytical solution, showing less than $3 \%$ relative errors, whereas the FD responses exhibit very large errors.

On the next step of the numerical study, we gradually increased the number of cells within the same FD modeling domain from a coarse grid to the fine one, by refining the areas surrounding the receivers, until the FD response was characterized by relative errors similar to those produced by the hybrid FD-IE method on the coarse grid. The fine grid, which was finally determined by this process, consisted of $81 \times 61 \times 52=256,932$ cells, with $100 \times$ $100 \mathrm{~m}$ uniform cell size in the $x$ - and $y$-directions, a minimum cell size of $5 \mathrm{~m}$ near the receiver positions, and a maximum cell size of $250 \mathrm{~m}$ in the $z$-direction, as shown in the right panel of Figure 1. Note that to have the level of errors for the FD responses similar to those for the hybrids FD-IE responses on the coarse grid, we had to refine not only the grid in the vicinity of the receivers, but also the entire grid within the FD modeling domain.

We ran the code using one node of a cluster with 20 Intel Xeon processors, $64 \mathrm{~GB}$, and $2.5 \mathrm{GHz}$. The computational time for the FD method on the coarse grid was approximately $10 \mathrm{~s}$. The hybrid FD-IE method required an additional time for Green's tensor calculation, and the total computational time was approximately $16 \mathrm{~s}$ (see Table 1). The FD method on the fine grid took approximately $196 \mathrm{~s}$. Therefore, to produce the same relative errors, as the hybrid FD-IE method on the coarse grid, the conventional FD algorithm had to be run on a fine grid, which required approximately 10 times as much computational time. Figures 2 and 3 present a 
comparison of the responses computed by the hybrid FD-IE method on the coarse grid, the FD method on the coarse and fine grids, and the 1D semianalytic solution based on the horizontally layered model with an isotropic resistive reservoir layer.

We have also computed the fields using the hybrid FD-IE method on the fine grid. The corresponding computational time and the relative errors are listed in Table 1. One can see that, if the grids are the same, the hybrid FD-IE method shows smaller relative errors than the FD method.

Thus, the modeling study for a simple horizontally layered model has demonstrated that the hybrid FD-IE method always provides the smaller errors than the conventional FD method for the same discretization grids. Also, the hybrid method provides practically the same responses with and without the mesh refinement near the receivers, whereas the results of the conventional FD method strongly depend on the mesh refinement, especially for the $\mathrm{E}_{z}^{\mathrm{a}}$ and $\mathrm{H}_{y}^{\mathrm{a}}$ fields.
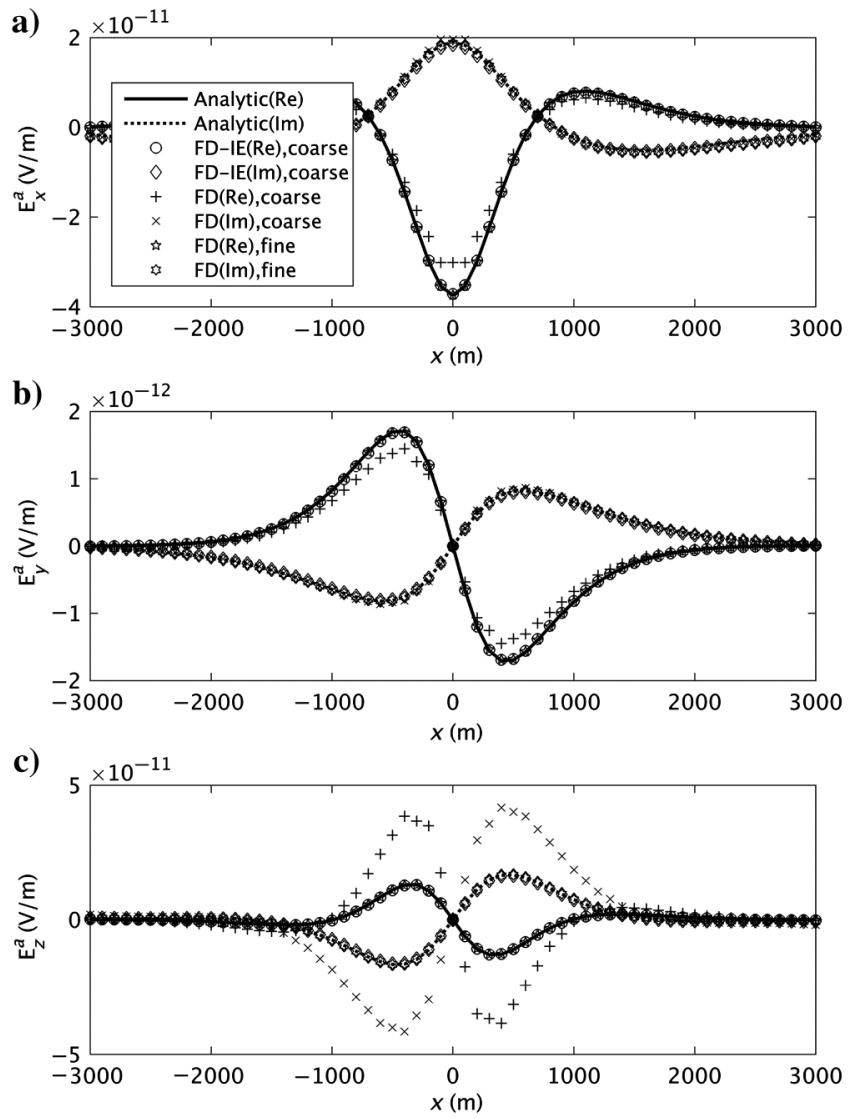

Figure 2. Model 1: The plots of anomalous electric fields computed using the hybrid FD-IE method, the FD method, and a 1D semianalytic solution based on the horizontally layered model with an isotropic resistive reservoir layer. The solid and dotted lines denote the real and imaginary parts of the fields computed by the 1D semianalytic solution. The real and imaginary parts of the fields computed by the hybrid FD-IE method on the coarse grid are denoted by circles and diamonds, respectively. The real and imaginary parts of the fields computed by the FD method on the coarse and fine grids are denoted by pluses, $x$-marks, pentagrams, and hexagrams, respectively. The panels $(\mathrm{a}-\mathrm{c})$ present the $x-, y$-, and $z$-components of the anomalous electric field, respectively.
It is clear that the current implementation of the hybrid scheme does not allow us to consider an anomalous layer of infinite extent due to the use of zero boundary condition for the anomalous field. In principle, this can be avoided by using a more accurate boundary condition, as was mentioned above. However, in our case studies, considered below, we are dealing with the anomalous domains having limited horizontal extent, which is typical for a hydrocarbon reservoir. In this situation, the current version of the hybrid scheme works well to resolve the target.

\section{Comparison with 3D IE method}

In the next model study, we have simulated the marine CSEM response of a 3D reservoir using the hybrid FD-IE scheme and compared the results with those produced by a conventional 3D IE method (Zhdanov et al., 2006).

We consider a 3D model with an anisotropic background and anisotropic 3D rectangular reservoir. The background is a sea-
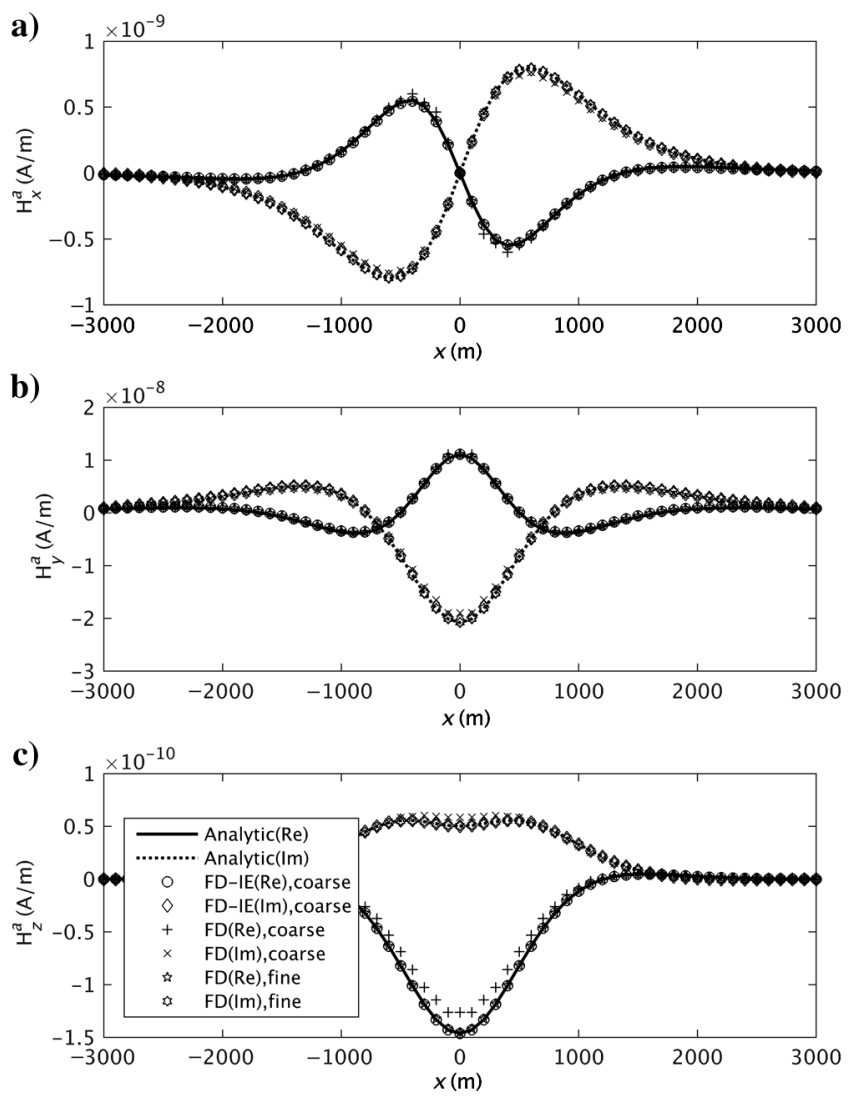

Figure 3. Model 1: The plots of anomalous magnetic fields computed using the hybrid FD-IE method, the FD method, and a 1D semi-analytic solution based on the horizontally layered model with an isotropic resistive reservoir layer. The solid and dotted lines denote the real and imaginary parts of the fields computed by the 1D semianalytic solution. The real and imaginary parts of the fields computed by the hybrid FD-IE method on the coarse grid are denoted by circles and diamonds, respectively. The real and imaginary parts of the fields computed by the FD method on the coarse and fine grids are denoted by pluses, x-marks, pentagrams, and hexagrams, respectively. The panels (a-c) present the $x$-, $y$-, and $z$-components of the anomalous magnetic field, respectively. 
water-sediments model consisting of three transverse anisotropic layers. From the first top sediment layer to the bottom halfspace, the horizontal resistivities are 1,2, and $4 \mathrm{ohm}-\mathrm{m}$, and the vertical resistivities are 5, 10, and $7 \mathrm{ohm}-\mathrm{m}$, respectively. The anisotropic resistive reservoir is embedded in the second sediment layer, with a horizontal resistivity of $10 \mathrm{ohm}-\mathrm{m}$ and a vertical resistivity of $100 \mathrm{ohm}-\mathrm{m}$ (Figure 4). The electric dipole oriented in the

Table 1. Model 1: Computational times and relative errors of the FD and hybrid FD-IE method for the horizontally layered model with the coarse and fine grids.

Coarse grid

Fine grid

\begin{tabular}{lrrrrrr} 
& Time (s) & \multicolumn{2}{c}{$\begin{array}{c}\text { Relative } \\
\text { error (\%) }\end{array}$} & Time (s) & \multicolumn{2}{c}{$\begin{array}{c}\text { Relative } \\
\text { error }(\%)\end{array}$} \\
\hline \multirow{2}{*}{ FD } & \multirow{2}{*}{10.28} & $\mathrm{E}_{x}$ & 15.63 & 196.19 & $\mathrm{E}_{x}$ & 3.20 \\
& & $\mathrm{E}_{y}$ & 15.28 & & $\mathrm{E}_{y}$ & 1.40 \\
& & $\mathrm{E}_{z}$ & 163.03 & & $\mathrm{E}_{z}$ & 1.57 \\
& & $\mathrm{H}_{x}$ & 6.47 & & $\mathrm{H}_{x}$ & 2.52 \\
& & $\mathrm{H}_{y}$ & 7.69 & & $\mathrm{H}_{y}$ & 2.93 \\
& & $\mathrm{H}_{z}$ & 13.21 & & $\mathrm{H}_{z}$ & 0.76 \\
Hybrid & $10.28+5.6$ & $\mathrm{E}_{x}$ & 3.01 & $196.19+20.82$ & $\mathrm{E}_{x}$ & 2.84 \\
FD-IE & & $\mathrm{E}_{y}$ & 1.59 & & $\mathrm{E}_{y}$ & 0.83 \\
& & $\mathrm{E}_{z}$ & 1.74 & & $\mathrm{E}_{z}$ & 0.95 \\
& & $\mathrm{H}_{x}$ & 2.02 & & $\mathrm{H}_{x}$ & 0.83 \\
& & $\mathrm{H}_{y}$ & 3.02 & & $\mathrm{H}_{y}$ & 2.86 \\
& & $\mathrm{H}_{z}$ & 0.19 & & $\mathrm{H}_{z}$ & 0.21 \\
& & & & & &
\end{tabular}

a)
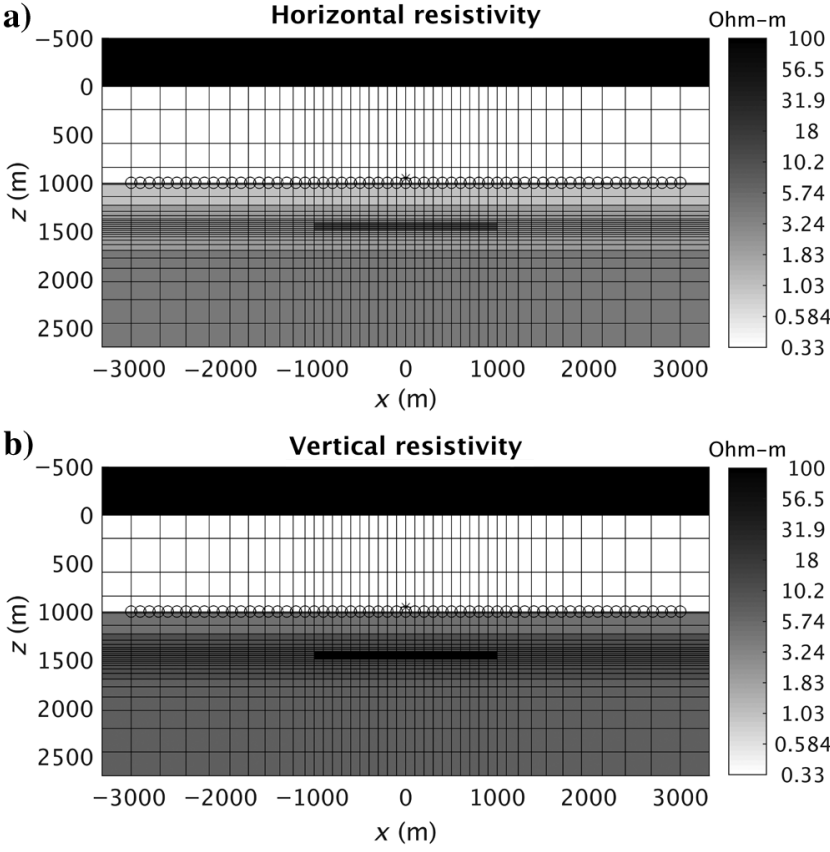

Figure 4. Model 2: Vertical sections of (a) the horizontal and (b) the vertical resistivity distributions. The star indicates the electric dipole source position, and the circles represent the receiver positions.
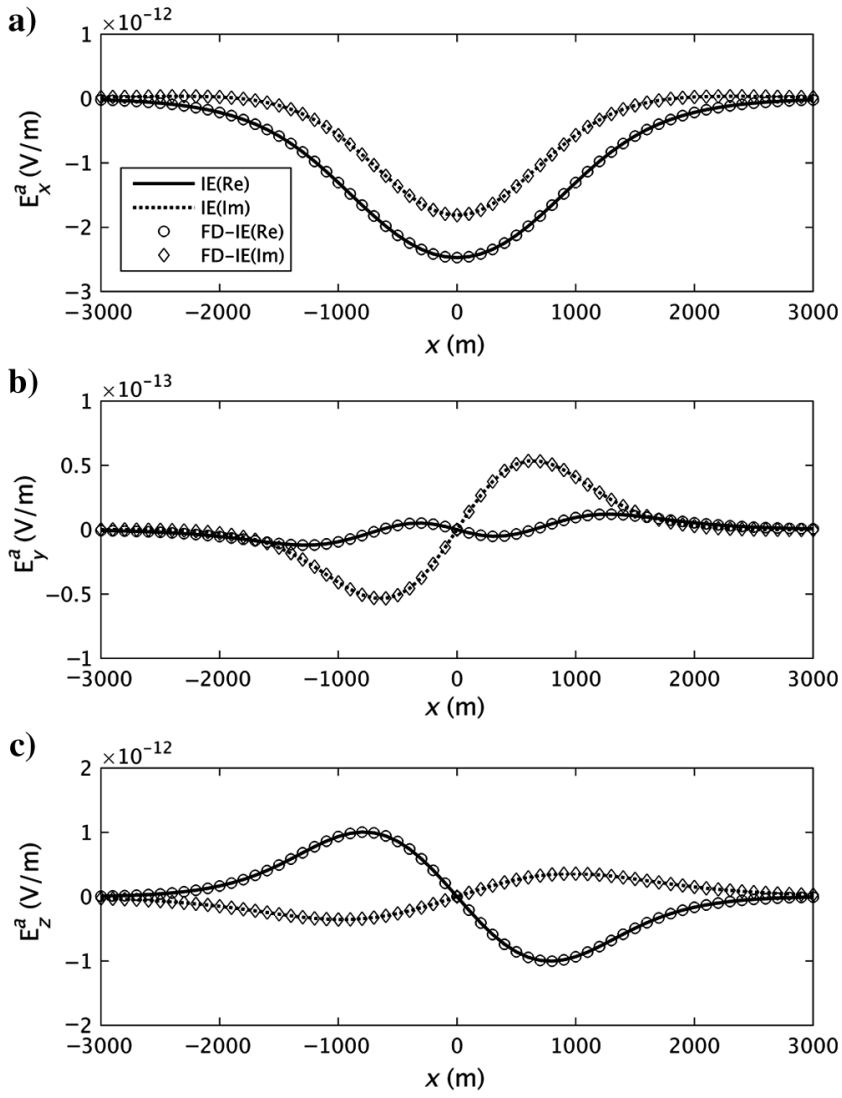

Figure 5. Model 2: A comparison of anomalous electric fields computed using the hybrid FD-IE method and the conventional IE method. The solid and dotted lines denote the real and imaginary parts of the fields computed by the IE method. The real and imaginary parts of the fields computed by the hybrid FD-IE method are denoted by circles and diamonds, respectively. The panels (a-c) present the $x$-, $y$-, and $z$-components of the anomalous electric field, respectively.

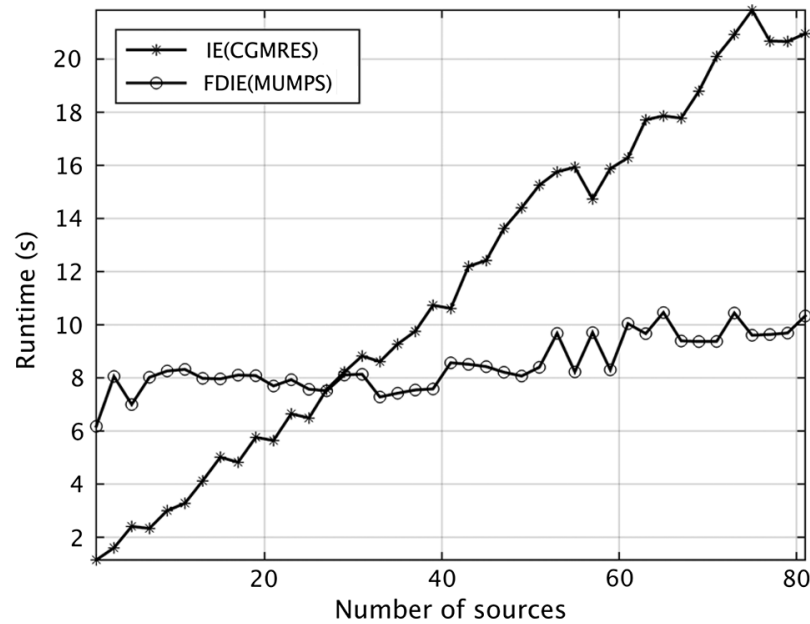

Figure 6. Model 2: Computation time of solving the corresponding systems of linear equations for the electric field within the anomalous domain for IE and hybrid FD-IE methods as a function of increasing number of the sources. 
$x$-direction is located in the seawater with coordinates $(0,0,950 \mathrm{~m})$, which is $50 \mathrm{~m}$ above the seafloor, and the frequency is $1 \mathrm{~Hz}$. The receivers are located $5 \mathrm{~m}$ above the seafloor from -3 to $3 \mathrm{~km}$ with $200 \mathrm{~m}$ spacing in the $x$-direction, and shifted them $50 \mathrm{~m}$ in the $y$-direction (at $y=-50 \mathrm{~m}$ ).

The FD modeling domain was selected as $\{-3 \leq x, y \leq 3 \mathrm{~km}$, $-0.5 \leq z \leq 3 \mathrm{~km}\}$ based on the skin depth, and it was discretized with a nonuniform grid, refined near the reservoir only, and not near the receivers. The reservoir (anomalous domain) was discretized using a $100 \times 100 \times 10 \mathrm{~m}$ uniform grid.

To demonstrate the accuracy and efficiency of the developed hybrid FD-IE method for 3D forward modeling, we have compared the FD-IE results with those calculated using a 3D IE method (Zhdanov et al., 2006). The PIE3D IE code is based on the parallel implementation of the contraction IE (CIE) method, and the system of the CIE equations is solved using an iterative solver, the complex generalized minimal residual (CGMRES) method (Zhdanov, 2002, 2009, 2015). In the framework of the IE method, we discretized the anomalous domain using the same cell size as those used within the anomalous domain in the hybrid FD-IE method. The IE and hybrid FD-IE codes were run on one node of a cluster with 20 Intel Xeon processors, $64 \mathrm{~GB}$, and $2.5 \mathrm{GHz}$. Figure 5 shows a comparison between the anomalous electric fields computed using the developed hybrid FD-IE method and the original IE method. One can see that the hybrid FD-IE method produces practically the same results as those obtained by the IE method.

We have also compared the computation times for both methods. The computations required by the both methods can be divided into two stages: (1) electric field calculation within the anomalous domain by solving the corresponding systems of linear equations and (2) calculation of the electric field at the receiver positions using the integral formulas in equation 3 . Note that stage 2 is identical for both methods. Therefore, we have only compared the computation times required for the first stage, which involves solving the corresponding systems of linear equations for the electric field within the anomalous domain. The root-mean-square misfit for the IE method, which uses an iterative solver, was set equal to $1^{-10}$, which was the misfit level achieved by the direct solver in the hybrid FD-IE method.

Figure 6 shows computation times of solving the corresponding systems of equations for both methods as a function of increasing number of the sources. As one can see, the IE method demonstrates an excellent performance if the number of the sources is relatively small (less than 25). Indeed, for a single source, the iterative solver CGMRES of the IE method took only approximately $1.5 \mathrm{~s}$, whereas the direct solver MUMPS of the hybrid FD-IE method required approximately $6.17 \mathrm{~s}$. However, if the number of sources increases, the computational time of the IE method increases linearly as well. At the same time, the runtime of the hybrid FD-IE method, practically, is not affected by the number of the sources. The reason is that the FD system of equations is solved using a MUMPS direct solver. This solver requires most of the computation time for numerical factorization and much less time for the forward and backward substitution, which depends on the number of sources (Chung et al., 2014).

These tests demonstrate that the developed hybrid FD-IE method provides a more accurate solution than the conventional FD method and, at the same time, it is characterized by a better performance than the conventional IE method for the multisource data.

\section{SYNTHETIC MODEL STUDY OF THE INVERSION ALGORITHM BASED ON THE HYBRID FD-IE METHOD}

We have applied our inversion algorithm to synthetic towed streamer EM data computer simulated for a typical sea-bottom geoelectrical model. Model 3 consists of two thin hydrocarbon reservoirs embedded in a conductive layer of the sea-bottom sediments as shown in Figure 7. The bodies at the left and right sides in Figure 7 represent oil and gas reservoirs with resistivities of 50 and $100 \mathrm{ohm}-\mathrm{m}$, respectively. The background consists of a $320 \mathrm{~m}$ seawater layer with a resistivity of 0.3 and $1 \mathrm{ohm}-\mathrm{m}$ half-space representing the sea-bottom sediments. The oil and gas reservoirs are located 1100 and $1200 \mathrm{~m}$ below the sea surface, with thicknesses of $200 \mathrm{~m}$ for both of them.

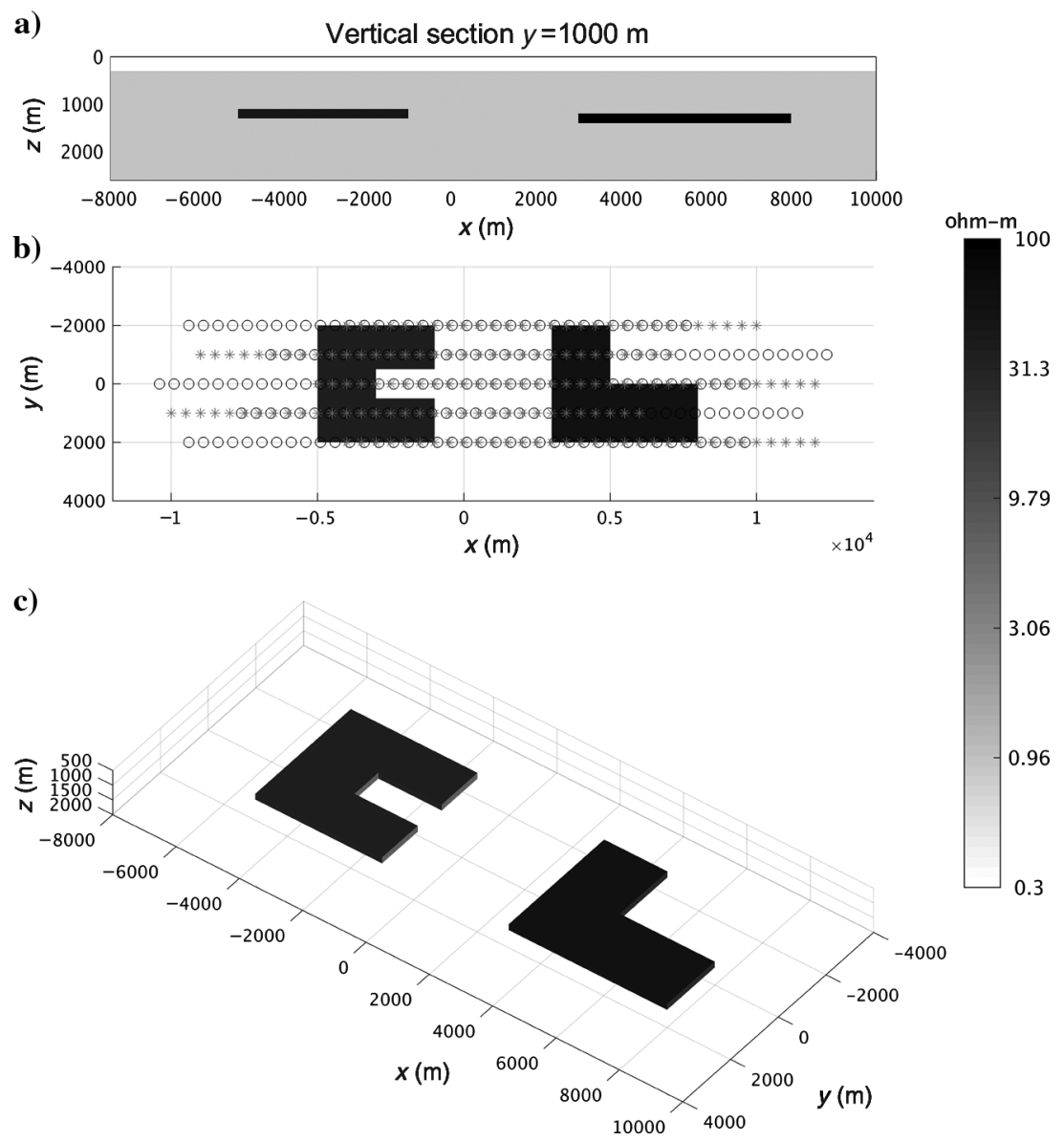

Figure 7. Model 3: (a) Vertical section at $y=1000 \mathrm{~m}$, (b) top view with transmitter (star) and receiver (circle) positions, and (c) 3D resistivity distributions. 
The EM survey consists of five survey lines running in the $x$-direction, with distances of $1 \mathrm{~km}$ between the lines as shown in Figure $7 \mathrm{~b}$. The electromagnetic field is excited at every $500 \mathrm{~m}$ by a horizontal electric dipole oriented in the $x$-direction with a moment of $1 \mathrm{Am}$, which is towed at a depth of $10 \mathrm{~m}$ below the sea surface. Five receivers with offsets between 2400 and $5400 \mathrm{~m}$ are towed at a depth of $100 \mathrm{~m}$ and measure inline electric fields at three frequencies between 0.1 and $2.75 \mathrm{~Hz}$. The synthetic observed towed streamer EM data were generated using the 3D IE forward modeling code (Hursán and Zhdanov, 2002) with cell sizes of $100 \times 100 \times 50 \mathrm{~m}$. The data were contaminated with random Gaussian noise of $2 \%$ of the total electric fields.

The dimensions of the inversion domain were $18 \times 8 \times 1.6 \mathrm{~km}$. The inversion domain was discretized into $250 \times 250 \times 100 \mathrm{~m}$ uniform cells, from a depth of 400 to $2000 \mathrm{~m}$. The FD modeling domain was designed by padding all sides of the inversion domain with four more layers with logarithmically increasing size; however, the discretization inside the modeling domain was kept the same as for the inversion domain. Note that different methods, domains, and discretizations were used for modeling and inversion. The regulari-
Figure 8 . The resistivity distribution recovered by the inversion of the synthetic towed streamer EM data on model 3. (a) Vertical section at $y=1000 \mathrm{~m}$, (b) horizontal section at a depth of $1300 \mathrm{~m}$, and (c) 3D resistivity distributions with a cutoff value of $3 \mathrm{ohm}-\mathrm{m}$. a)

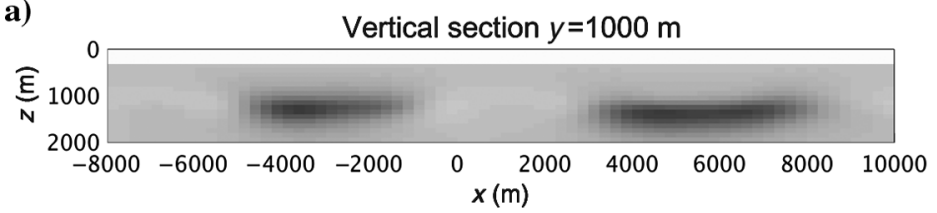

b)
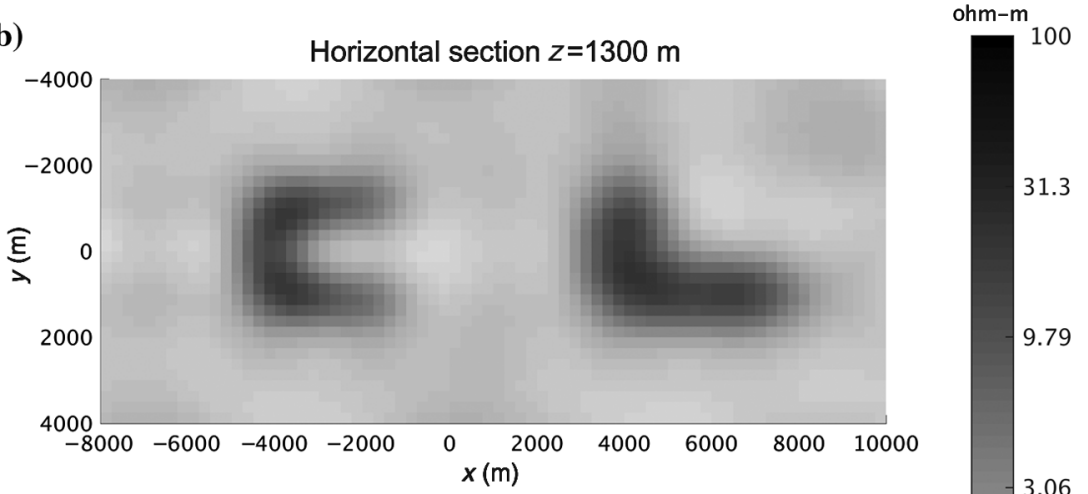

c)
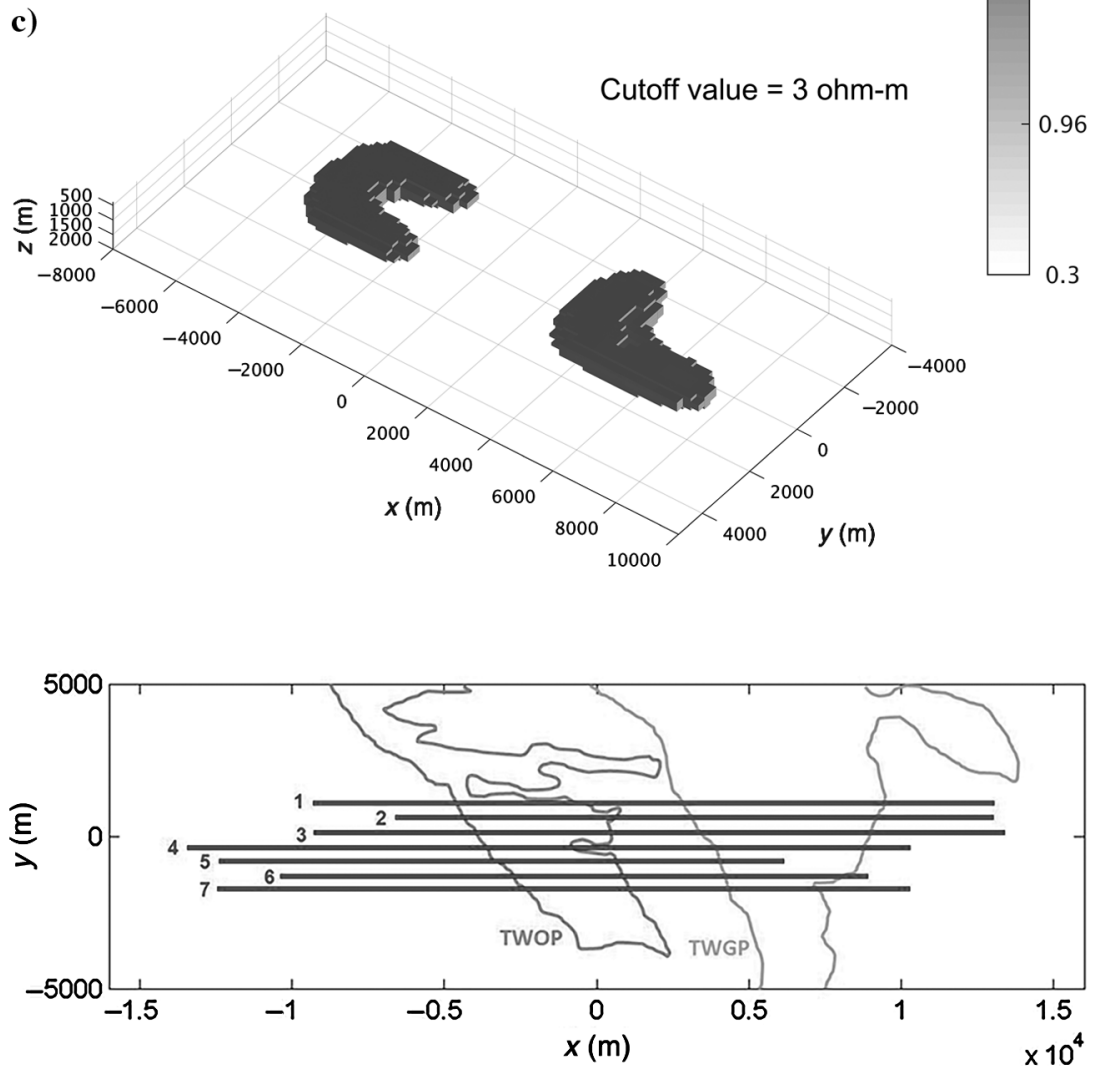

Figure 9. The survey configuration for seven-line data inversion in the local coordinate system. 
zation was based on the combination of the minimum norm and minimum vertical support stabilizing functional. The inversions were run on one node of a cluster with 20 Intel Xeon processors, $64 \mathrm{~GB}$, and $2.5 \mathrm{GHz}$, and it required approximately 3 hours and 25 iterations until the misfit between the observed and predicted data reached the noise level of $2 \%$.

Figure 8 presents the inversion results. As one can see from the results, the inversion recovered well the shapes and depths of the reservoirs.

\section{D INVERSION OF TOWED STREAMER EM DATA COLLECTED FROM THE TWOP}

We applied the 3D inversion based on the hybrid FD-IE method to towed streamer EM data collected in the TWOP. The Troll field is located in the Norwegian sector in the northern part of the North Sea. The field is separated into three parts: the TWOP, the Troll West Gas Province (TWGP), and the Troll East Gas Province. The towed streamer EM data were acquired by PGS over the Troll

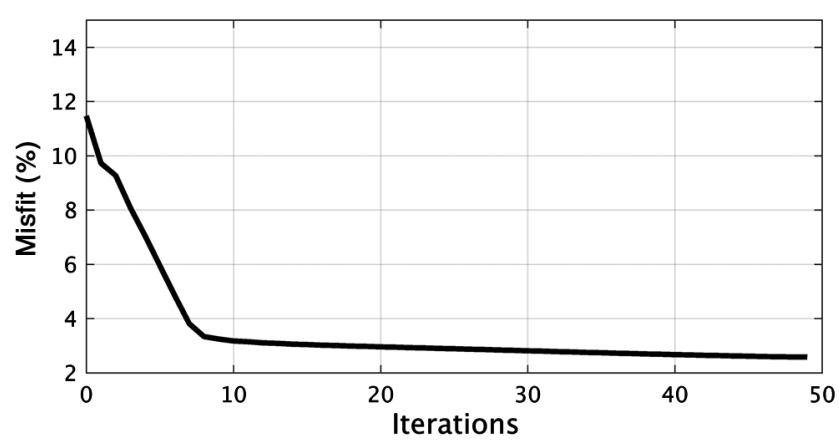

Figure 10. Convergence plot for hybrid FD-IE inversion of the Troll survey data. field in 2010 and 2012, and the data were inverted successfully based on the IE method (Zhdanov et al., 2014a, 2014b). In the current paper, considering the size constraints of the journal publication, we present the results of the isotropic inversion only using the data acquired in 2012. However, the hybrid FD-IE could be applied for an anisotropic inversion as well. For more information about the data, geologic setting, and exploration history, we refer the reader to Zhdanov et al. (2014a, 2014b).

The towed streamer EM data we inverted comprised seven lines of data at three frequencies between 0.1 and $1.04 \mathrm{~Hz}$. The $8700 \mathrm{~m}$ long EM streamer with eleven receivers with offsets between 1860 and $7554 \mathrm{~m}$ was towed at $100 \mathrm{~m}$ depth. The EM source was towed at $10 \mathrm{~m}$ depth. Figure 9 shows the survey configuration for the sevenline data over the true locations of the TWOP and TWGP in the local coordinate system. In this case study, the inversion domain represents the entire volume under the survey area, where we would like to reconstruct a $3 \mathrm{D}$ distribution of the conductivity. The inversion domain was selected from -16 to $16 \mathrm{~km},-4$ to $4 \mathrm{~km}$, and 400 to $2400 \mathrm{~m}$ in the $x$-, $y$-, and $z$-directions. The inversion domain was discretized using a uniform grid with a cell size of $250 \times 250 \times 50 \mathrm{~m}$. The horizontal size of the MSD was $4 \times 4 \mathrm{~km}$. The starting model for the inversion consists of $320 \mathrm{~m}$ seawater with a resistivity of 0.27 and a 2 ohm-m half-space. The inversion was run without any a priori information. The process of iterative inversion was terminated after 49 iterations when the normalized misfit reached $2.5 \%$. Figure 10 shows the convergence plot for the hybrid FD-IE inversion of the Troll survey data.

We present in Figure 11 a comparison of the observed and predicted data for the final inversion result along line 1 . Note that, we present in this figure the conventional common middle point (CMP) plots with the half of actual offsets shown in the vertical axis. One can see a very good fit between the real and the imaginary parts of the observed towed streamer electric field data and the synthetic data, generated for the inverse model at a frequency of $0.496 \mathrm{~Hz}$ with the exception of the very near-offset data. This can be caused a)

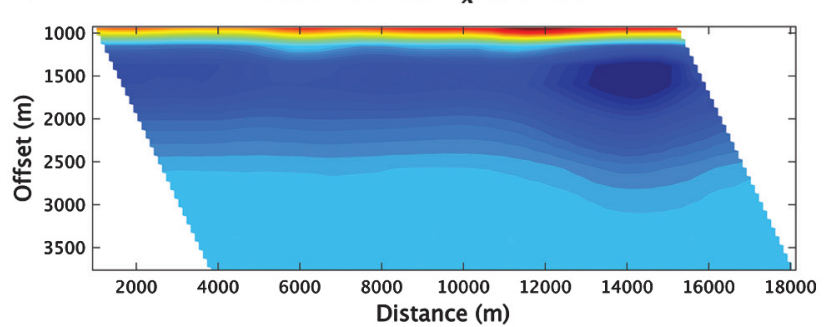

b)

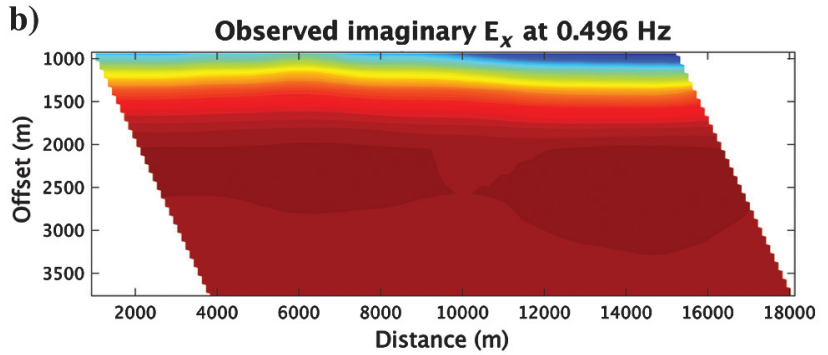

c)

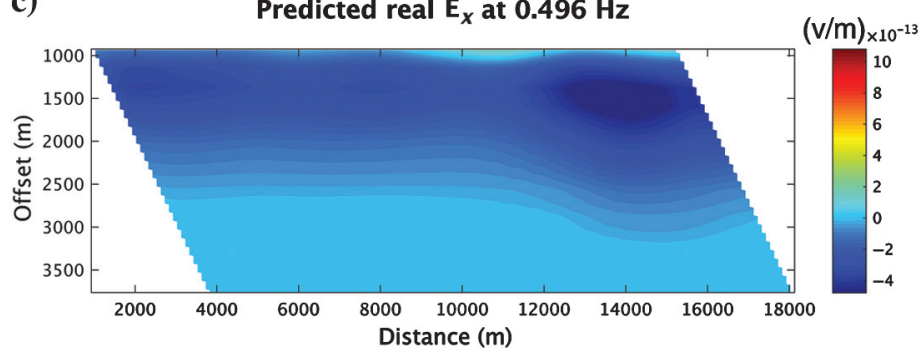

d)

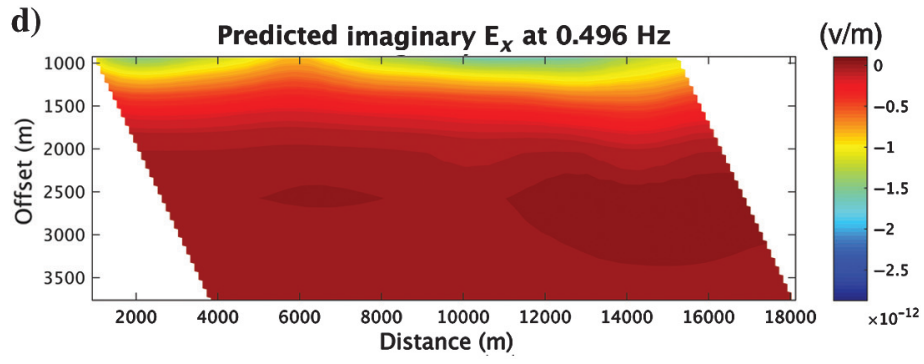

Figure 11. (a and b) Observed and (c and d) predicted real, (a and c) and (b and d) imaginary data for inline electric fields at $0.496 \mathrm{~Hz}$ along line 1 presented as the CMP plots for the case of unconstraint hybrid FD-IE inversion of Troll field data. 
by simplifications used in our inversion by assuming constant seawater conductivity and flat bathymetry.

Figure 12 presents the inversion results at this misfit level. As one can see, the inversion results correlate very well with the true positions of the TWOP and TWGP reservoirs. Also, the recovered images agree well with those recovered by Zhdanov et al. (2014b) using fully parallelized IE-based inversion.

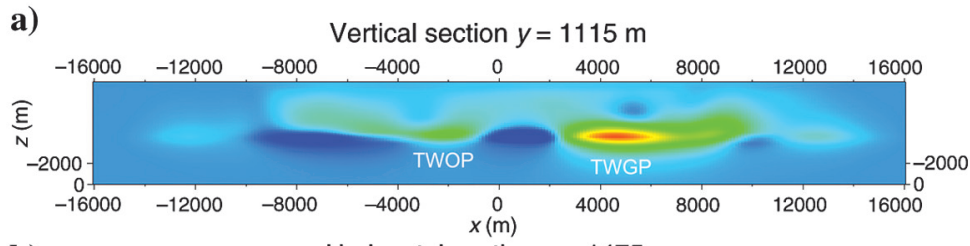

b)
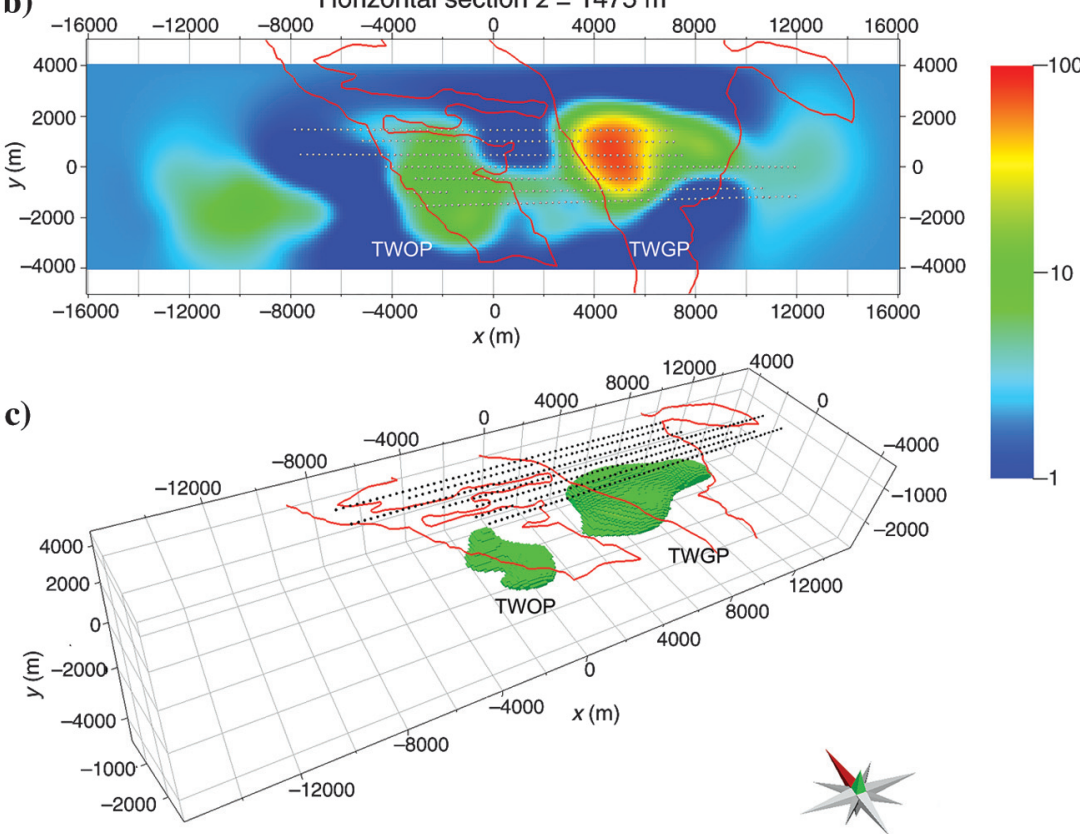

Figure 12. The resistivity distribution recovered by the inversion of the towed streamer EM data from Troll field. (a) Vertical section at $y=1115 \mathrm{~m}$ below the survey line 1 , (b) horizontal section at a depth of $1475 \mathrm{~m}$ (white dots represent transmitter positions), and (c) 3D resistivity distributions with a cutoff value of $8 \mathrm{ohm}-\mathrm{m}$.

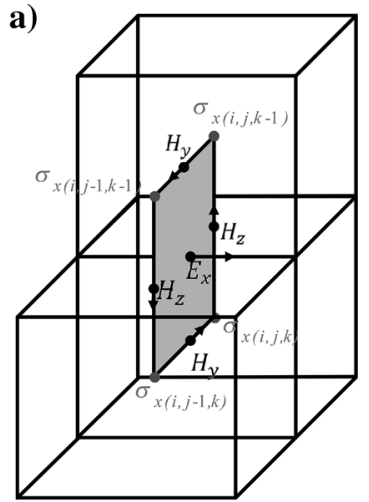

b)
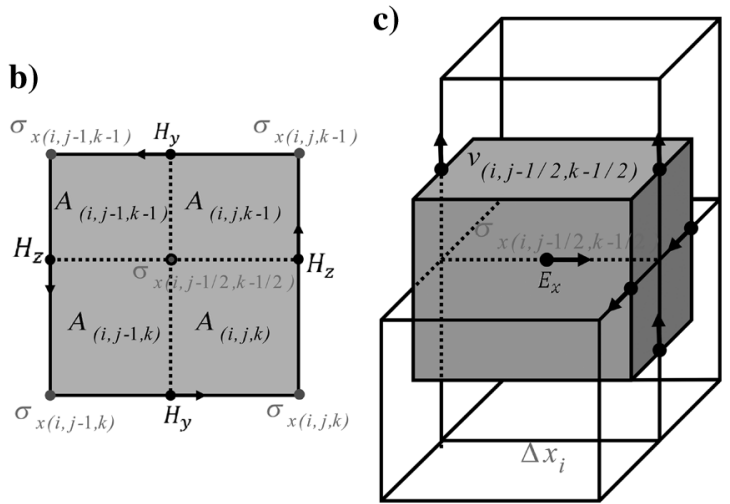

Figure A-1. Illustration of the process of computing an average conductivity and the corresponding volume.

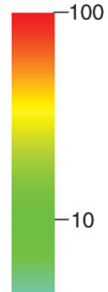

\section{CONCLUSIONS}

We have developed a novel 3D modeling and inversion approach, combines the advantages of the FD and IE methods. In the frame The corresponding system of linear FD equations is solved using a direct solver, MUMPS, which is based on a multifrontal method for LU factorization. After the unknown electric fields are determined in the modeling domain using the FD method, the electric and magnetic fields at the receiver are computed based on the Green's tensor approach. This approach makes it possible to compute the fields at the receivers accurately without the need of very fine FD discretization in the vicinity of the receivers and without the need for numerical differentiation and interpolation. The hybrid FD-IE modeling method was carefully validated by comparing the results with a $1 \mathrm{D}$ semianalytical solution and with the $3 \mathrm{D}$ IE solution. We have also developed an algorithm of 3D inversion of towed streamer EM data based on the novel hybrid FD-IE method. The developed inversion method was demonstrated in synthetic model study and a case study from the Troll field. The resistivity images produced by the hybrid FD-IE inversion method agree well with the known reservoir extents and previous inversion results for the Troll field towed streamer EM data.

\section{ACKNOWLEDGMENTS}

The authors acknowledge TechnoImaging, the University of Utah's Consortium for Electromagnetic Modeling and Inversion and Petroleum Geo-Services for support of this research and permission to publish. We also thank M. Čuma for helping us to set up the direct solver MUMPS.

\section{APPENDIX A}

\section{WEIGHTED AVERAGING CONDUC- TIVITY AND THE CORRESPONDING VOLUME}

In the staggered-grid scheme, the anomalous electric fields are assigned to the edges of the cells. The conductivities where the electric fields are located are represented by a weighted average of conductivities of the four adjoining cells based on Ampere's law (Wang and Hohmann, 1993; Alumbaugh et al., 1996).

For example, the $x$-directional conductivity $\Delta \sigma_{x(i, j-1 / 2, k-1 / 2)}$ located at the edge-center is averaged by the areas of the four adjoining cells (Figure A-1a and A-1b) as follows: 


$$
\begin{aligned}
& \Delta \sigma_{x(i, j-1 / 2, k-1 / 2)} \\
& =\frac{\Delta \sigma_{x(i, j, k)} A_{(i, j, k)}+\Delta \sigma_{x(i, j-1, k)} A_{(i, j-1, k)}+\Delta \sigma_{x(i, j, k-1)} A_{(i, j, k-1)}+\Delta \sigma_{x(i, j-1, k-1)} A_{(i, j-1, k-1)}}{A_{(i, j, k)}+A_{(i, j-1, k)}+A_{(i, j, k-1)}+A_{(i, j-1, k-1)}} .
\end{aligned}
$$

The volume for the corresponding auxiliary cell with respect to the averaged conductivity $\Delta \sigma_{x(i, j-1 / 2, k-1 / 2)}$ is calculated (Figure A-1c) as follows:

$$
\begin{aligned}
v_{(i, j-1 / 2, k-1 / 2)}= & \left(A_{(i, j, k)}+A_{(i, j-1, k)}+A_{(i, j, k-1)}\right. \\
& \left.+A_{(i, j-1, k-1)}\right) \cdot \Delta x_{i},
\end{aligned}
$$

where $\Delta x_{i}$ is the length of the corresponding edge, which determines the dimension of the cell in the $x$-direction. Similar considerations are valid for the $y$ - and $z$-components of the average conductivities and the corresponding volumes. This volume of the auxiliary cell is used to solve the volume integral of Green's function in equation 10 based on the Gaussian quadrature method.

\section{APPENDIX B}

\section{CALCULATION OF FRÉCHET DERIVATIVES}

To derive the Fréchet derivatives based on the QB approximation on the staggered grid, we start with a discrete form of the equation 12 at inversion iteration $(n)$ as follows:

$$
\begin{aligned}
& \mathrm{E}_{\alpha}^{\mathrm{a}(n)}\left(r_{l}\right) \\
& =\sum_{i=1}^{N_{x}} \sum_{j=1}^{N_{y}+1} \sum_{k=1}^{N_{z}+1} \Gamma_{\alpha x}^{l(i, j-1 / 2, k-1 / 2)} \Delta \sigma_{x(i, j-1 / 2, k-1 / 2)}^{(n)} \mathrm{E}_{x(i, j-1 / 2, k-1 / 2)}^{(n)} \\
& +\sum_{i=1}^{N_{x}+1} \sum_{j=1}^{N_{y}} \sum_{k=1}^{N_{z}+1} \Gamma_{\alpha y}^{l(i-1 / 2, j, k-1 / 2)} \Delta \sigma_{y(i-1 / 2, j, k-1 / 2)}^{(n)} \mathrm{E}_{y(i-1 / 2, j, k-1 / 2)}^{(n)} \\
& +\sum_{i=1}^{N_{x}+1} \sum_{j=1}^{N_{y}+1} \sum_{k=1}^{N_{z}} \Gamma_{\alpha z}^{l(i-1 / 2, j-1 / 2, k)} \Delta \sigma_{z(i-1 / 2, j-1 / 2, k)}^{(n)} \mathrm{E}_{z(i-1 / 2, j-1 / 2, k)}^{(n)},
\end{aligned}
$$

where $\mathrm{E}_{\alpha}^{\mathrm{a}(n)}\left(r_{l}\right)$ is the $\alpha$ component of a predicted anomalous electric field at the receiver position; $r_{l}, \Delta \sigma_{\beta}^{(n)}$, and $\mathrm{E}_{\beta}^{(n)}$ are anomalous conductivity and electric fields in the $\beta$ direction within the inversion domain on the staggered grid.

By substituting equation A-1 into equation B-1, we can find the Fréchet derivatives based on QB approximation with respect to the $\beta$ directional conductivity $\Delta \sigma_{\beta}$. For example, the Fréchet derivative of the $\alpha$ component of the electric fields, receiver position $r_{l}$, and $n$ iteration with respect to the $x$-directional conductivity $\Delta \sigma_{x(i, j, k)}^{(n)}$ is

$$
\begin{aligned}
& \left.\mathrm{F}_{\alpha}^{(n)}\left(r_{l}\right)\right|_{\Delta \sigma_{x(i, j, k)}} \\
& =F_{\alpha x}^{(n)}\left(r_{l}\right)=\frac{A_{(i, j, k)} \Gamma_{\alpha x}^{l(i, j-1 / 2, k-1 / 2)} \mathrm{E}_{x(i, j-1 / 2, k-1 / 2)}^{(n)}}{A_{(i, j, k)}+A_{(i, j-1, k)}+A_{(i, j, k-1)}+A_{(i, j-1, k-1)}} \\
& +\frac{A_{(i, j, k)} \Gamma_{\alpha x}^{l(i, j+1 / 2, k-1 / 2)} \mathrm{E}_{x(i, j+1 / 2, k-1 / 2)}^{(n)}}{A_{(i, j, k)}+A_{(i, j+1, k)}+A_{(i, j, k-1)}+A_{(i, j+1, k-1)}} \\
& +\frac{A_{(i, j, k)} \Gamma_{\alpha x}^{l(i, j-1 / 2, k+1 / 2)} \mathrm{E}_{x(i, j-1 / 2, k+1 / 2)}^{(n)}}{A_{(i, j, k)}+A_{(i, j-1, k)}+A_{(i, j, k+1)}+A_{(i, j-1, k+1)}} \\
& +\frac{A_{(i, j, k)} \Gamma_{\alpha x}^{l(i, j+1 / 2, k+1 / 2)} \mathrm{E}_{x(i, j+1 / 2, k+1 / 2)}^{(n)}}{A_{(i, j, k)}+A_{(i, j+1, k)}+A_{(i, j, k+1)}+A_{(i, j+1, k+1)}}
\end{aligned}
$$

In the case of a cell-center-based grid such as in the IE method, the Fréchet derivative based on QB approximation with respect to $\Delta \sigma_{x(i, j, k)}^{(n)}$ can be represented as follows:

$$
\left.\mathrm{F}_{\alpha}^{(n)}\left(r_{l}\right)\right|_{\Delta \sigma_{x(i, j, k)}}=\Gamma_{\alpha x}^{l(i, j, k)} \mathrm{E}_{x(i, j, k)}^{(n)} .
$$

Comparing equations B-2 and B-3, one can see that the Fréchet derivative at a point $(i, j, k)$ is a weighted average of the Fréchet derivatives at the four points of the edge-centers of the cell $(i, j, k)$. The Fréchet derivatives with respect to the $y$ - and $z$-directional conductivity can be derived in a similar way.

In matrix notation, equation B-2 can be generalized as follows:

$$
\mathbf{F}_{\beta}^{(n)}=\left.\mathbf{F}^{(n)}\right|_{\Delta \sigma_{\beta}}=\hat{\mathbf{G}}_{\beta}^{\prime \mathrm{E}} \mathbf{E}_{\beta}^{\prime(n)} \mathbf{C}_{\beta}=\left[\begin{array}{c}
\mathbf{G}_{x \beta}^{\prime \mathrm{E}} \\
\mathbf{G}_{y \beta}^{\mathrm{IE}} \\
\mathbf{G}_{z \beta}^{\prime \mathrm{E}}
\end{array}\right] \mathbf{E}_{\beta}^{\prime(n)} \mathbf{C}_{\beta},
$$

where $\mathbf{F}_{\beta}^{(n)}$ is a $3 L \times N$ matrix of the Fréchet derivatives with respect to the $\beta$ directional conductivity, $\Delta \boldsymbol{\sigma}_{\beta} ; \hat{\mathbf{G}}_{\beta}^{\prime \mathrm{E}}$ is a $3 L \times N_{\beta}^{e}$ matrix containing the electric Green's tensor integrals with respect to the $\beta$ directional conductivity; $\mathbf{E}_{\beta}^{\prime(n)}$ is an $N_{\beta}^{e} \times N_{\beta}^{e}$ diagonal matrix with $\beta$ components of the electric fields; and $\mathbf{C}_{\beta}$ is an $N_{\beta}^{e} \times N$ weighted averaging matrix. For representation purposes, we introduce the weighted averaging matrix $\mathbf{C}_{\beta}$ but the actual calculation of the weighted average of $\hat{\mathbf{G}}_{\beta}^{\prime \mathrm{E}} \mathbf{E}_{\beta}^{\prime(n)}$ can be easily made in a $3 \mathrm{D}$ matrix array.

In the case of isotropy, the Fréchet matrix can be represented as

$$
\begin{aligned}
\left.\mathbf{F}^{(n)}\right|_{\Delta \sigma}= & {\left[\begin{array}{l}
\mathbf{G}_{x x}^{\prime \mathrm{E}} \\
\mathbf{G}_{y x}^{\prime \mathrm{E}} \\
\mathbf{G}_{z x}^{\prime \mathrm{E}}
\end{array}\right] \mathbf{E}_{x}^{\prime(n)} \mathbf{C}_{x}+\left[\begin{array}{c}
\mathbf{G}_{x y}^{\prime \mathrm{E}} \\
\mathbf{G}_{y y}^{\prime \mathrm{E}} \\
\mathbf{G}_{z y}^{\prime \mathrm{E}}
\end{array}\right] \mathbf{E}_{y}^{\prime(n)} \mathbf{C}_{y} } \\
& +\left[\begin{array}{c}
\mathbf{G}_{x z}^{\prime \mathrm{E}} \\
\mathbf{G}_{y z}^{\prime \mathrm{E}} \\
\mathbf{G}_{z z}^{\prime \mathrm{E}}
\end{array}\right] \mathbf{E}_{z}^{\prime(n)} \mathbf{C}_{z}=\mathbf{F}_{x}^{(n)}+\mathbf{F}_{y}^{(n)}+\mathbf{F}_{z}^{(n)} .
\end{aligned}
$$

The Fréchet derivatives for the magnetic field can be found by replacing the electric Green's tensors in equation B-5 with the magnetic Green's tensors.

As one can see, the Green's tensors used for field calculations in equation B-1 are the same as those used for Fréchet calculation in equation B-2. Therefore, we can precompute the Green's tensors on 
the staggered grid only once and reuse them for the Fréchet calculation as well as the field calculation, which results in a very efficient inversion method.

\section{REFERENCES}

Abubakar, A., M. Li, G. Pan, J. Liu, and T. M. Habashy, 2011, Joint MT and CSEM data inversion using a multiplicative cost function approach: Geophysics, 76, no. 3, F203-F214, doi: 10.1190/1.3560898.

Alumbaugh, D. L., G. A. Newman, L. Prevost, and J. N. Shadid, 1996, Three dimensional wideband electromagnetic modeling on massively parallel computers: Radio Science, 31, 1-23, doi: 10.1029/95RS02815.

Amestoy, P. R., I. S. Duff, J.-Y. L'Excellent, and J. Koster, 2001, A fully asynchronous multifrontal solver using distributed dynamic scheduling: SIAM Journal on Matrix Analysis and Application, 23, 15-41, doi: 10 $.1137 / \mathrm{S} 0895479899358194$.

Amestoy, P. R., A. Guermouche, J.-Y. L'Excellent, and S. Pralet, 2006, Hybrid scheduling for the parallel solution of linear systems: Parallel Computing, 32, 136-156, doi: 10.1016/j.parco.2005.07.004.

Avdeev, D. B., 2005, Three-dimensional electromagnetic modeling and inversion from theory to application: Surveys in Geophysics, 26, 767-799, doi: $10.1007 / \mathrm{s} 10712-005-1836-\mathrm{x}$.

Avdeev, D. B., and S. Knizhnik, 2009, 3D integral equation modeling with a linear dependence on dimensions: Geophysics, 74, no. 5, F89-F94, doi: 10.1190/1.3190132.

Cox, L. H., G. A. Wilson, and M. S. Zhdanov, 2012, 3D inversion of airborne electromagnetic data: Geophysics, 77, no 4, WB59-WB69, doi: 10 .1190/GEO2011-0370.1.

Cox, L. H., and M. S. Zhdanov, 2014, 3D airborne electromagnetic inversion using a hybrid edge-based FE-IE method with moving sensitivity domain: 84th Annual International Meeting, SEG, Expanded Abstracts, 739-744.

Chung, Y., J. S. Son, T. J. Lee, H. J. Kim, and C. Shin, 2014, Three-dimensional modeling of controlled-source electromagnetic surveys using an edge finite-element method with a direct solver: Geophysical Prospecting, 62, 1468-1483, doi: 10.1111/gpr.2014.62.issue-6..

da Silva, N. V., J. V. Morgan, L. MacGregor, and M. Warner, 2012, A finite element multifrontal method for 3D CSEM modeling in the frequency domain: Geophysics, 77, no. 2, E101-E115, doi: 10.1190/ geo2010-0398.1.

Endo, M., M. Cuma, and M. S. Zhdanov, 2008, A multigrid integral equation method for large-scale models with inhomogeneous backgrounds: Journal of Geophysics and Engineering, 5, 438-447, doi: 10.1088/1742-2132/5/ $4 / 007$.

Grayver, A. V., R. Streich, and O. Ritter, 2013, Three-dimensional parallel distributed inversion of CSEM using a direct forward solver: Geophysical Journal International, 193, 1432-1446, doi: 10.1093/gii/ggt055.

Gribenko, A., and M. S. Zhdanov, 2007, Rigorous 3D inversion of marine CSEM data based on the integral equation method: Geophysics, $\mathbf{7 2}$, no. 2, WA73-WA84, doi: 10.1190/1.2435712.

Hursán, G., and M. S. Zhdanov, 2002, Contraction integral equation method in three-dimensional electromagnetic modeling: Radio Science, 37, 1-11-13, doi: $10.1029 / 2001$ RS002513.

Jaysaval, P., D. Shantsev, and S. K. Ryhove, 2014, Fast multimodel finitedifference controlled-source electromagnetic simulations based on a Schur complement approach: Geophysics, 79, no. 6, E315-E327, doi: 10.1190/geo2014-0043.1.

Newman, G. A., and D. L. Alumbaugh, 1995, Frequency-domain modelling of airborne electromagnetic responses using staggered finite differences:
Geophysical Prospecting, 43, 1021-1042, doi: 10.1111/gpr.1995.43. issue-8.

Newman, G.A., 2014, A review of high-performance computational strategies for modeling and imaging of electromagnetic induction data: Surveys in Geophysics, 35, 85-100, doi: 10.1007/s10712-013-9260-0.

Schwarzbach, C., and E. Haber, 2013, Finite element based inversion for time-harmonic electromagnetic problems: Geophysical Journal International, 193, 615-634, doi: 10.1093/gji/ggt006.

Streich, R., 2009, 3D finite-difference frequency-domain modeling of controlled-source electromagnetic data: Direct solution and optimization for high accuracy: Geophysics, 74, no. 5, F95-F105, doi: 10.1190/1 .3196241

Tikhonov, A. N., and V. Y. Arsenin, 1977, Solution of ill-posed problems: V. H. Winston.

Wang, T., and G. W. Hohmann, 1993, A finite difference time-domain solution for three-dimensional electromagnetic modeling: Geophysics, $\mathbf{5 8}$, 797-809, doi: 10.1190/1.1443465.

Ward, S. H., and G. W. Hohmann, 1988, Electromagnetic theory for geophysical applications, in M.N. Nabighian, ed., Electromagnetic methods in applied geophysics 1: Investigations in Geophysics, 130-311.

Yang, D., and D. W. Oldenburg, 2012, Three-dimensional inversion of airborne time-domain electromagnetic data with applications to a porphyry deposit: Geophysics, 77, no. 2, B23-B34, doi: 10.1190/ geo2011-0194.1.

Yee, K., 1966, Numerical solution of initial boundary value problems involving Maxwell's equations in isotropic media: IEEE Transactions on Antennas Propagation, 14, 302-307, doi: 10.1109/TAP.1966 .1138693 .

Zaslavsky, M., V. Druskin, S. Davydycheva, L. Knizhnerman, A. Abubakar, and T. Habashy, 2011, Hybrid finite-difference integral equation solver for 3D frequency domain anisotropic electromagnetic problems: Geophysics, 76, no. 2, F123-F137, doi: 10.1190/1.3552595.

Zhdanov, M. S., and G. Keller, 1994, The geoelectrical methods in geophysical exploration: Elsevier.

Zhdanov, M. S., 2002, Geophysical inverse theory and regularization problems: Elsevier.

Zhdanov, M. S., S. K. Lee, and K. Yoshioka, 2006, Integral equation method for 3D modeling of electromagnetic fields in complex structures with inhomogeneous background conductivity: Geophysics, 71, no. 6, G333-G345, doi: 10.1190/1.2358403.

Zhdanov, M. S., 2009, Geophysical electromagnetic theory and methods: Elsevier.

Zhdanov, M. S., and L. Cox, 2012, Method of subsurface imaging using superposition of sensor sensitivities from geophysical data acquisition systems: U. S. Patent US 2013/0173163.

Zhdanov, M. S., L. Cox, and J. Rudd, 2013, Paradigm change in 3D inversion of airborne EM surveys: Case study for oil sands exploration near Fort McMurray, Alberta: First Break, 31, 81-85.

Zhdanov, M. S., M. Endo, L. H. Cox, M. Čuma, J. Linfoot, C. Anderson, N. Black, and A. V. Gribenko, 2014a, Three dimensional inversion of towed streamer electromagnetic data: Geophysical Prospecting, 62, 552-572, doi: 10.1111/gpr.2014.62.issue-3 1365-2478.12097.

Zhdanov, M. S., M. Endo, D. Yoon, C. Čuma, J. Mattsson, and J. Midgley, 2014b, Anisotropic 3D inversion of towed-streamer electromagnetic data: Case study from the Troll West oil province: Interpretation, 2, no. 3, SH97-SH113, doi: 10.1190/INT-2013-0156.1.

Zhdanov, M. S., 2015, Inverse theory and applications in geophysics: Elsevier. 